\title{
Efficient Estimation of Parameters of the Extreme Value Distribution
}

\author{
by
}

Sathi Rani Saha

A Thesis submitted to the Faculty of Graduate Studies of The University of Manitoba in partial fulfilment of the requirements for the degree of

MASTER OF SCIENCE

Department of Statistics

University of Manitoba

Winnipeg

Copyright (C) 2014 by Sathi Rani Saha 


\begin{abstract}
The problem of efficient estimation of the parameters of the extreme value distribution has not been addressed in the literature. We obtain efficient estimators of the parameters of type I (maximum) extreme value distribution without solving the likelihood equations. This research provides for the first time simple expressions for the elements of the information matrix for type II censoring. We construct efficient estimators of the parameters using linear combinations of order statistics of a random sample drawn from the population. We derive explicit formulas for the information matrix for this problem for type II censoring and construct efficient estimators of the parameters using linear combinations of available order statistics with additional weights to the smallest and largest order statistics. We consider numerical examples to illustrate the applications of the estimators. We also perform an extensive Monte Carlo simulation study to examine the performance of the estimators for different sample sizes.
\end{abstract}




\section{Acknowledgments}

I would like to exprese my sincere gratitude to my thesis advisor Professor Saumen Mandal for his valuable guidence and support from the begining of this research and writing the thesis. It would have been fairly impossible to conduct this research and prepare this thesis without his close supervision. I would also like to thank Professor Mrityunjay Samanta for his brilliant remarks and suggestions. I extend my heartfelt appreciation to my thesis committee members Professor A. Thavaneswaran and Professor R. Thulasiram for their critiques and valuable comments.

I must be thankful to the Department of Statistics, the Faculty of Science and the Faculty of Graduate Studies for giving the financial support to carry out my research.

Finally, I express my sincere appreciation to my family members whose love, support, encouragement and sacrifice help me to face problems confidently in every stage of my life. 


\section{Dedication}

Dedicated to my beloved son Soptom 


\section{Contents}

Contents

List of Tables $\quad$ iv

1 Introduction 1

2 Estimation of the parameters of the extreme value distribution on the basis of a complete sample $\quad 12$

3 Estimation of the parameters of the extreme value distribution on the basis of a censored sample

4 Estimation of $\theta$ in the model $\frac{1}{c \theta} f\left(\frac{x-\theta}{c \theta}\right)$ on the basis of complete and censored samples

5 Conclusions 


\section{List of Tables}

3.1 Values of the infinite sums $(-1) S(p)$ and $(-1) S(1-p)$ for different values of $p \ldots \ldots \ldots \ldots \ldots \ldots \ldots$

3.2 Elements of the matrix $\mathcal{I}^{*}=\left(\left(\mathcal{I}_{i j}^{*}\right)\right)_{2 \times 2} \ldots \ldots \ldots \ldots$

3.3 Elements of the matrix $\mathcal{I}^{*-1}=\left(\left(\mathcal{I}^{* i j}\right)\right)_{2 \times 2} \ldots \ldots \ldots$. . . . .

3.4 Means, variances and covariances of the proposed estimators of parameters of type I (maximum) extreme value distribution $(\theta=0$, $\sigma=1$ ) from 100000 samples of size $n=10 \ldots \ldots \ldots$. . . . . . 51

3.5 Means, variances and covariances of the proposed estimators of parameters of type I (maximum) extreme value distribution $(\theta=0$, $\sigma=1$ ) from 100000 samples of size $n=16 \ldots \ldots$. . . . . . .

3.6 Means, variances and covariances of the proposed estimators of parameters of type I (maximum) extreme value distribution $(\theta=0$, $\sigma=1$ ) from 100000 samples of size $n=20 \ldots \ldots$. . . . . . .

3.7 Means, variances and covariances of the proposed estimators of parameters of type I (maximum) extreme value distribution $(\theta=0$, $\sigma=1$ ) from 100000 samples of size $n=24 \ldots \ldots \ldots$ 
3.8 Means, variances and covariances of the proposed estimators of parameters of type I (maximum) extreme value distribution $(\theta=0$, $\sigma=1$ ) from 100000 samples of size $n=28 \ldots \ldots \ldots \ldots$

3.9 Means, variances and covariances of the proposed estimators of parameters of type I (maximum) extreme value distribution $(\theta=0$, $\sigma=1$ ) from 100000 samples of size $n=30 \ldots \ldots \ldots$ 


\section{Chapter 1}

\section{Introduction}

In statistics, there are two types of estimation: point and interval estimation. Here we deal with the point estimation. Point estimation calculates a single value using the sample data to estimate an unknown population parameter $\theta$. The estimation of $\theta$ is done through a statistic. Suppose that we have a random sample of observations $X_{1}, X_{2}, \ldots, X_{n}$. A point estimator is a function $T\left(X_{1}, X_{2}, \ldots . ., X_{n}\right)$ of a sample; that is, a statistic is a point estimator (Casella and Berger (2002)). The rationale behind point estimation is quiet simple. Following an example may help to understand the use of estimation. Suppose the experimenter wants to know the average height of students of the Departments of Statistics of the University of Manitoba. Using all students is time consuming and costly. An experimenter draws some samples from popualation and take the sample mean to make conclusion about the average height of all the students. This sample mean is a point estimator of the population mean. There are several methods of finding estimators: Method of Moments, Least Squares Estimation, Maximum Likelihood Estimation, Bayes estimation, and the EM algorithm. After finding the estimators a question arises, how "good" they are? To answer this question we need to study some properties of estimators. 
One of the properties of estimator is unbiasedness. Gauss (1821) introduced the concept of unbiasedness property. According to Gauss, the unbiasedness peoperty of an estimator is considered as very attractive (Mukhopadhyay (1950)). Mathematically, a real valued statistic $\mathrm{T}=T\left(X_{1}, X_{2}, \ldots, X_{n}\right)$ is called an unbised estimator of $\theta$ if and only if $E_{\theta}(T)=\theta$ for all $\theta \in \Theta$ where $\Theta$ is the parameter space . In other words, the distribution of $T$ should be centered about the true value of $\theta$. The bias of an estimator is written as: $\operatorname{Bias}(\hat{\theta})=E(\hat{\theta})-\theta$ where $\hat{\theta}$ is an estimator of $\theta$. In practice, there could be more than one unbiased estimator of a parameter. The "best" estimator is chosen using some appropiate criteria.

Mean square error is an important property for comparing two estimators. Suppose that the real valued statistic $\mathrm{T}=T\left(X_{1}, X_{2}, \ldots, X_{n}\right)$ is an estimator of $\theta$. Then, the mean square error (MSE) of the estimator $\mathrm{T}$ is given by $E_{\theta}\left[(T-\theta)^{2}\right]$. If $\mathrm{T}$ is unbiased for $\theta$, MSE is the variance of $\mathrm{T}$, denoted by $V_{\theta}(T)$. Among all unbiased estimators, estimator with the smallest variance is preferred.

In estimation theory, there could be more than one unbiased estimator and the good estimator is seleted using the principle of minimum variance. An unbiased estimator $\mathrm{T}(\mathrm{X})$ of $\theta$ is called UMVUE (Uniformly Minimum Variance Unbiased Estimator) if and only if (i) $E_{\theta}(T)=\theta \forall \theta$ (ii) $\operatorname{var}_{\theta}(T(X)) \leq \operatorname{var}_{\theta}\left(T^{\prime}(X)\right) \quad \forall \theta$ and for all unbiased estimators $T^{\prime}$ of $\theta$. In other words, an estimator is UMVUE if it is unbiased and if there is no other unbiased estimator with smaller variance for all $\theta \in \Theta$. In terms of MSE (Mean Square Error), UMVUE has the smallest variance among all unbiased estimators.

Another important property of an estimator is consistency. This is a large sample 
property. It describes the limiting behaviour of the estimator $\hat{\theta}$ when the sample size tends to infinity. In 1922, R. A. Fisher introduced the concept of consistency. An estimator is said to be consistent if the difference between the parameter and the estimator goes to zero when the sample size tends to infinity. Mathematically, an estimator $\mathrm{T}$ of the parameter $\theta$ is called consistent, if $\mathrm{T}$ converges to $\theta$ in probability, that is, $\lim _{n \rightarrow \infty} P[|T-\theta| \leq \epsilon]=1$ (Bartoszynski and Niewiadomska-Bugaj (2009)). This means, when the sample size gets larger the sample information becomes better and better and the estimator approaches arbitrarily close to the parameter value.

Another property of an estimation is sufficiency. The objective of the use of sufficient statistics is that it condences the data in such a way that no information about $\theta$ is lost. Sufficient statistic provides maximum information about the parameter $\theta$. In other words, sufficient statistics represent or summarize all information in a random sample for estimating the unknown parameter $\theta$. Mathematically, a statistic $\mathrm{T}(\mathrm{X})$ is a sufficient statistic for $\theta$ if the conditional distribution of the sample $\mathrm{X}$ given the value of $\mathrm{T}(\mathrm{X})$ does not depend on $\theta$ (Casella and Berger (2002)). Neyman-Factorization theorem is useful for the identification of a sufficient statistic. A sufficient statistic $\mathrm{T}(\mathrm{X})$ is called a minimal sufficient statistic if, for any other sufficient statistic $T^{\prime}(X), \mathrm{T}(\mathrm{X})$ is a function of $T^{\prime}(X)$ (Casella and Berger (2002)). This implies that the minimal sufficient statistic utilizes the minimal set of points to represent the sample data. In order to find the minimal sufficient statistic, Lehmann-Scheffe's Minimal Sufficiency Theorem is useful.

Another property of an estimator is completeness. It guarantees the uniqueness of an unbiased estimator which may be a function of sufficient statistics. This estimator becomes UMVUE automaticaly. Mathematically, let $f(t ; \theta)$ be a family of p.d.f.s for 
a statsitic $T(\mathbf{X})$. The family of distributions is called complete, iff $E_{\theta}(g(T))=0 ; \forall \theta$ which implies that $P_{\theta}(g(T)=0)=1$ for all $\theta$. That is, if $E(g(T))=0 \forall \theta$ then $g(T)=0$ for almost everywhere. $T(\mathbf{X})$ is called a complete statistic.

Efficiency is the large sample property of an estimator. The term efficiency is concerned with the asymptotic variance of an estimator. Let $\tau(\theta)$ be a real valued function of $\theta$. A sequence of estimators $T_{n}$ is asymptotically efficient for $\tau(\theta)$ if $\sqrt{n}\left[T_{n}-\tau(\theta)\right] \rightarrow[0, v(\theta)]$ in distribution and $v(\theta)=\frac{\left[\tau^{\prime}(\theta)\right]^{2}}{n E_{\theta}\left[\left(\frac{\partial}{\partial \theta}[\log f(X ; \theta)]\right)^{2}\right]}$. That is, the asymptotic variance of $T_{n}$ achives the Cramer-Rao Lower Bound (Casella and Berger (2002)). Fisher information and Cramer-Rao Inequality are described below.

Fisher information plays an important role in estimation theory. We define the Fisher information $I(\theta)$ in the random sample $\mathrm{X}$ as: $I(\theta)=E_{\theta}\left[l^{\prime}(X \mid \theta)\right]^{2}$ where $l(x \mid \theta)=\log f(x \mid \theta)$ is the $\log$-likelihood function, and $l^{\prime}(x \mid \theta)=\frac{\partial}{\partial \theta} \log f(x \mid \theta)=\frac{f^{\prime}(x \mid \theta)}{f(x \mid \theta)}$ where $f^{\prime}(x \mid \theta)$ is the derivative of $f(x \mid \theta)$ with respect to $\theta$ (Rao (1973)). For computational purposes, Fisher information can also be written as

$$
\begin{aligned}
I(\theta) & =-E_{\theta}\left[l^{\prime \prime}(X \mid \theta)\right] \\
& =-E_{\theta}\left(\frac{\partial^{2}}{\partial \theta^{2}} \log f(x \mid \theta)\right) .
\end{aligned}
$$

Suppose that $\mathrm{T}=\mathrm{T}(\mathrm{X})$ is an unbiased estimator of a real valued parametric function $\tau(\theta)$, that is, $E_{\theta}(T)=\tau(\theta)$ for all $\theta \in \Theta$. Then, for all $\theta \in \Theta$, under the standard assumptions, we have,

$$
\operatorname{Var}_{\theta}(T) \geq \frac{\left[\tau^{\prime}(\theta)\right]^{2}}{n E_{\theta}\left[\left(\frac{\partial}{\partial \theta}[\log f(X ; \theta)]\right)^{2}\right]}
$$


This inequality is known as Cramer-Rao inequality and is also known as information inequality. During the 1940s Indian statistician C. R. Rao and Swedish Statistician H. Cramer developed this inequality. When $\tau(\theta)=\theta$, the inequality becomes, $\operatorname{Var}_{\theta}(T) \geq \frac{1}{n I(\theta)}$ where

$$
I(\theta)=E_{\theta}\left[\left(\frac{\partial}{\partial \theta}[\log f(X ; \theta)]\right)^{2}\right]
$$

$I(\theta)$ is called the Fisher Information. When $I(\theta)$ increases, the variance of the estimator decreases. Therefore the quality of the estimator increases. For an i.i.d. sample of size n, no other unbiased estimators of the parameter have a variance smaller than Cramer-Rao Lower Bound (CRLB). An unbiased estimator is said to be efficient if it attains this lower bound. In addition, an estimator with this lower bound is also called the UMVUE.

In this thesis, we focus on efficient estimation of parameters of the extreme value distribution. The question of efficient estimation of parameters of extreme value distribution has not been addressed in the literature. First we discuss the extreme value distributions and their uses. Extreme value theory deals with the extreme (rare) events which have very small probability to occur. Examples of rare events are: extreme temperature, extreme flood and snowfalls, large fluctuations in exchange rates, high wind speeds and market crashes. In order to assess the risks and to develop models, engineers and business analysts use the extreme value distribution. Extreme value distributions are the limiting distributions for the minimum and the maximum values of a sample obtained from independent and identically distributed random variables. Extreme value distributions are very important in statistics. Along with generalized extreme value distribution these models play an important role 
in risk management, finance, insurance, hydrology, telecommunications, economics, material sciences and many other industries dealing with extreme values.

There are three types of Extreme Value Distributions (EVD's): types I, II and III. The extreme value type I is also known as the Gumbel distribution. This distribution is named by a German mathematician Emil Gumbel (1891-1966). Type I distribution has two forms: one is for minimum cases and the other is based on the maximum cases. The pdf of Gumbel distribution is (for minimum): $f(x)=$ $\frac{1}{\beta} e^{\frac{x-\mu}{\beta}} e^{-e^{\frac{x-\mu}{\beta}}}, x \in \mathbf{R} ; \mu$ is the location parameter and $\beta$ is the scale parameter. When $\mu=0$ and $\beta=1$, this reduces to standard Gumbel distribution whose p.d.f. is given by $f(x)=e^{x} e^{-e^{x}}, x \in \mathbf{R}$. The pdf of Gumbel distribution is (for maximum): $f(x)=\frac{1}{\beta} e^{-\frac{x-\mu}{\beta}} e^{-e^{\frac{x-\mu}{\beta}}}, x \in \mathbf{R} ; \mu$ is the location parameter and $\beta$ is the scale parameter. When $\mu=0$ and $\beta=1$, this reduces to standard Gumbel distribution and is given by $f(x)=e^{-x} e^{-e^{x}}, x \in \mathbf{R}$. Some properties of Extreme value Type I distribution (for maximum) are: 1) distribution function: 1- $e^{-e^{-\frac{x-\mu}{\beta}}} \cdot 2$ ) Hazard function: $\frac{e^{[-(x-\mu) / \beta]}}{\beta\left(e^{e[-(x-\mu) / \beta]}-1\right)}$. 3) Moment generating function: $e^{\mu t} \Gamma(1-\beta t), t<1 / \beta$. 4) Mean: $\mu-\beta \Gamma^{\prime}(1)$. 5) Variance : $\beta^{2} \pi^{2} / 6$ (Haan and Rerreira (2006)). Gumbel distribution is widely used in Hydrology to analyze monthly and annual values of daily rainfall, river discharge volumes and the droughts. It is also useful to analyze the extreme earthquake, flood or any other natural disasters.

Extreme value type II distribution is also known as Frechet distribution. French mathematician Maurice Frechet (1878-1973) introduced this limiting distribution.The 
pdf of extreme value type II distribution is defined by

$$
f(x)=\frac{\alpha}{\beta}\left(\frac{\beta}{x-\gamma}\right)^{\alpha+1} e^{-\left(\frac{\beta}{x-\gamma}\right)^{\alpha}}, \gamma<x<+\infty
$$

where $\alpha$ is the shape parameter $(\alpha>0), \beta$ is the scale parameter $(\beta>0)$ and $\gamma$ is the location parameter. Note that $\gamma \equiv 0$ reduces to two-parameter Frechet distribution. This is the limiting distribution for a sequence of maxima. Frechet distribution is used in finance and adequate modeling of market-returns. However, Frechet distribution is relatively used less than Gumbel distribution.

Extreme value type III distribution for minimum values is known as Weibull distribution. Swedish engineer and scientist Waloddi Weibull (1887-1979) was the pioneer of Extreme value type III distribution. The pdf of two-parameter Weibull distribution is given by: $f(x)=\frac{\alpha}{\beta}\left(\frac{x}{\beta}\right)^{\alpha-1} e^{-\left(\frac{x}{\beta}\right)^{\alpha}}$; where $\alpha$ is the shape parameter $(\alpha>1)$, and $\beta$ is the scale parameter $(\beta>0)$. Weibull distribution was mainly developed to address the problems for minima (in material sciences) but not for maxima. However, becasue of its flexibility it is widely used in many other areas. When $\alpha=1$ it reduces to the exponencial distribution. When $\alpha=2$ it becomes the Rayleigh distribution which is used in telecommunications.

The Generalized extreme value (GEV) distribution is also knwon as Fisher-Tippet distribution. It is a three parameter model that combines the Gumbel, Frechet and Weibull maximum extreme value distributions. This is the limiting distribution of maxima of a sequence of independent and identically distributed random variables. The pdf is given by

$$
f(x)=\frac{1}{\sigma} t(x)^{\xi+1} e^{-t(x)}
$$


where

$$
t(x)= \begin{cases}\left(1+\left(\frac{x-\mu}{\sigma}\right) \xi\right)^{-1 / \xi} & \text { if } \xi \neq 0 \\ e^{-(x-\mu) / \sigma} & \text { if } \xi=0\end{cases}
$$

where $\xi, \sigma$ and $\mu$ are the tail shape, scale, and location parameters respectively. Different values of shape parameter produce the extreme value type I, II and III distribution. Specifically, $\xi=0, \xi>0$ and $\xi<0$ correspond, respectively to the Gumbel, Frechet and Reversed Weibull distribution. When $\xi=0$, GEV distribution reduces to the Gumbel class which includes the normal, gamma, exponencial, and lognormal distributions. If $\xi>0$, the GEV belongs to Frechet distribution and this includes well known Cauchy, Pareto and Student's t distributions. Finally, the distributions associated with $\xi<0$, are called weibull class including uniform and beta distributions. GEV has great importance in hydrology. It is widely used for modeling extremes of natural phenomena.

We now focus on the type I (maximum) extreme value distribution. Suppose $X_{1}, X_{2}, \ldots, X_{n}$ are independent and identically distributed random variables having the pdf of a type I (maximum) extreme value distribution

$$
g(x ; \theta, \sigma)=\frac{1}{\sigma} f\left(\frac{x-\theta}{\sigma}\right)
$$

where

$$
f(y)=\exp \left(-y-e^{-y}\right),-\infty<y<\infty
$$

and $\theta$ and $\sigma$ are unknown location and scale parameters respectively $(-\infty<\theta<$ $\infty, 0<\sigma<\infty)$. The estimation and testing hypothesis about $\theta$ and $\sigma$ on the basis 
of complete and censored samples have a fairly long history (Johnson et al. (1995) and Kotz and Nadarajah (2000)). For more work on the subject we refer to Chernoff et al. (1967), Chen (1998), Wu and Li (2003), Broussard (1998), Koutsoyiannis (2004) and Balakrishnan et al. (2004). In 1966, Downton (1966) found linear estimates of the parameter of the extreme value distribution for complete samples. Prescott and Walden (1980) developed maximum likelihood estimation of the parameters of the generalized extreme value distribution. Hosking et al. (1985) derived estimators of parameters of generalized extreme value distribution using the method of probability weighted moments. The use of order statistics in estimating the parameters of extreme value distribution was considered by Lieblein (1953). He developed explicit formula for the $k^{\text {th }}$ moment of the single order statistics for $n \leq 6$ for both complete and censored data. For samples up to size 20, Hassanein (1969) derived the best linear unbised estimator of the location and scale parameter based on two or three order statistics. Estimation of the parameters by sample quantiles has been judged by Hassanein (1968), Hassanein (1972). Raynal and Salas (1986) presented six estimation methods for the extreme value type-I distribution and compared by using data generation techniques. According to them the best linear combination of order statistics compares positively with the other methods for samples of sizes $\mathrm{n} \leq 20$. Lloyd (1952) developed least squares estimation of location and scale parameters using order statistics, resulting estimates being unbiased, linear in the ordered observations and of minimal variance. Kimball (1956) worked on bias of the maximum likelihood estimate of extreme value distribution and obtained the explicit formula to obtain the bias. Hosking (1984) carried out a study to test whether the shape parameter is zero in the generalized extreme value distribution. 
Bhoj (1997) estimated the location and scale parameters of extreme value distribution using ranked set sampling procedure. He compared these estimates with the ordered least squares estimates and showed that the relative precisions of these estimators are higher than those of ordered least squares estimators. Balakrishnan and Chan (1992b) worked on extreme value distribution for finding the means, variances and covariances of the order statistics. They also derived the best linear unbised estimators of location and scale parameters of extreme value distribution for various choices of censoring (Balakrishnan and Chan (1992a)). Fard and Holmquist (2008) provided explicit expressions for variances and covariances for order statistics from the standard extreme value distributions which seems complicated and troublesome to handle. However according to them, the approximation presented for variances and covariances for order statistics is easy to use and gives good approximate values. Fard and Holmquist (2007) presented approximate expressions of the first moment of the order statistics of standard extreme value distributions and they showed that the approximations give values with good precision.

For more work we refer to Hosking (1984), Haan and Resnick (1993), Deheuvels and Oliveira (1989), Einmahl et al. (1993), Tawn (1988), Balcerak (2011), and Galambos (1978). It follows from the previous works on estimating extreme value distribtuion that solving maximum likelihood function of extreme value distribution is complicated. The beauty of this thesis is to find the simple expression of the information matrix without solving the maximum likelihood functions.

Extreme value distributions are generally used to model the smallest or largest value in a large set of independent, and identically distributed random values representing measurements or observations. The extreme value distribution is 
appropriate for describing the distribution of the smallest observations in repeated random samples drawn from a distribution whose tails decay exponentially fast. In many practical situations, the extreme value distribution can be used as an appropriate statistical model. The oldest problems connected with extreme value distribution arise from floods. Problems related to extreme value distribution are also found in quality control, population statistics, economics, astronomy, meteorology, naval engineering, oceanography, and in connection with breaking strength of material and building code. As an example, suppose that there are 30 big hospitals in a country. In a given year, we record the largest weight of all new-born babies in each hospital. It is then possible to model those 30 largest weights using an extreme value distribution.

We discuss estimation of parameters $(\theta, \sigma)$ of the extreme value distribution based on the complete sample in Chapter 2. In Chapter 3, we discuss estimating the parameters $(\theta, \sigma)$ in the case of type II censoring and derive the expressions for the observed Fisher Information. The results of an extensive simulation study conducted to evaluate the performance of our estimators are also provided in Chapter 3. In Chapter 4 , we construct an efficient estimator of the parameter $\theta$ in the reduced model $\frac{1}{c \theta} f\left(\frac{x-\theta}{c \theta}\right)$ where $\theta(>0)$ is unknown and $c(>0)$ is known for complete and censored samples. A numerical example is used for illustration in each chapter. Finally, we give some concluding remarks in Chapter 5. 


\section{Chapter 2}

\section{Estimation of the parameters of the extreme value distribution on the basis of a complete sample}

Parts of the contents in the following chapters also appear in Saha et al. (2014) to which I contributed as the first author with Dr. M. Samanta and Dr. S. Mandal.

In this chapter we find the efficient estimator of parameters of extreme value distribution in the case of complete samples. The Fisher information matrix is used to calculate the variance covarience matrix. We consider the problem of estimation

of $\theta$ and $\sigma$ in the model $\frac{1}{\sigma} f\left(\frac{x-\theta}{\sigma}\right)$ when $f$ is the standard form of the pdf of type I (maximum) extreme value distribution. We construct efficient estimators of $\theta$ and $\sigma$ using linear combinations of order statistics of a random sample drawn from the population.

Here we consider an estimator $\left(T_{1 n}, T_{2 n}\right)$ of $(\theta, \sigma)$ of the form

$$
T_{i n}=n^{-1} \sum_{j=1}^{n} J_{i}\left(\frac{j}{n+1}\right) X_{j n}, \quad i=1,2
$$


where $X_{1 n} \leq X_{2 n} \leq \ldots \leq X_{n n}$ are the ordered observations and $J_{i}, i=1,2$ are well-behaved weight functions. This type of L-estimators (estimators using linear combinations of order statistics) has been studied extensively by Daniell (1920), Bennett (1952), Chernoff and Savage (1958), Chernoff et al. (1967), Bickel (1973) and David. and Nagaraja (2003), among others. Following Chernoff et al. (1967) we obtain explicit formulas for the weight functions $J_{i}, i=1,2$ and show that $\left(T_{1 n}, T_{2 n}\right)$ is asymptotically normally distributed with mean vector $(\theta, \sigma)$ and covariance matrix equal to $\sigma^{2}(n \mathcal{I})^{-1}$ where $\mathcal{I}$ is the Fisher information matrix.

We note that the random variable $Y=\frac{X_{1}-\theta}{\sigma}$ has pdf $f(y),-\infty<y<\infty$. Following Mandal and Samanta (2007) and Mandal and Samanta (2006) we define functions $L_{1}(y)$ and $L_{2}(y)$ by

$$
L_{1}(y)=-\frac{f^{\prime}(y)}{f(y)} \quad \text { and } \quad L_{2}(y)=-\left\{1+y \frac{f^{\prime}(y)}{f(y)}\right\}
$$


Then the Fisher information matrix is given by $\sigma^{-2} \mathcal{I}$ where $\mathcal{I}=\left(\left(\mathcal{I}_{i j}\right)\right)_{2 \times 2}$ and

$$
\begin{aligned}
& \mathcal{I}_{11}=\mathbf{E}\left\{L_{1}^{2}(Y)\right\} \\
& \mathcal{I}_{12}=\mathbf{E}\left\{L_{1}(Y) L_{2}(Y)\right\}, \quad\left[\mathcal{I}_{12}=\mathcal{I}_{21}\right] \\
& \mathcal{I}_{22}=E\left\{L_{2}^{2}(Y)\right\} .
\end{aligned}
$$

Now, $L_{1}(y)=-\frac{f^{\prime}(y)}{f(y)}$

$$
\begin{aligned}
f(y) & =e^{-y} e^{-e^{-y}} \\
f^{\prime}(y) & =e^{-y} e^{-e^{-y}} e^{-y}-e^{-e^{-y}} e^{-y}=e^{-2 y} e^{-e^{-y}}-e^{-y} e^{-e^{-y}} \\
f^{\prime \prime}(y) & =e^{-2 y} e^{-e^{-y}} e^{-y}-2 e^{-2 y} e^{-e^{-y}}-e^{-y} e^{-e^{-y}} e^{-y}+e^{-y} e^{-e^{-y}} \\
& =e^{-3 y} e^{-e^{-y}}-2 e^{-2 y} e^{-e^{-y}}-e^{-2 y} e^{-e^{-y}}+e^{-y} e^{-e^{-y}} \\
& =e^{-3 y} e^{-e^{-y}}-3 e^{-2 y} e^{-e^{-y}}+e^{-y} e^{-e^{-y}} \\
L_{1}(y) & =-\frac{e^{-y} e^{-e^{-y}}\left[e^{-y}-1\right]}{e^{-y} e^{-e^{-y}}} \\
& =1-e^{-y} \\
L_{2}(y) & =-\left\{1+y \frac{f^{\prime}(y)}{f(y)}\right\} \\
& =-\left\{1+y\left(-1+e^{-y}\right\}\right. \\
& =-1+y-y e^{-y} .
\end{aligned}
$$

Now we derive the elements of Fisher information matrix. 


$$
\begin{array}{rl}
\mathcal{I}_{11}= & \mathbf{E}\left\{L_{1}^{2}(Y)\right\} \\
= & \left.\mathbf{E}\left(1-e^{-y}\right)^{2} \quad \text { using equation in }(2.4)\right] \\
= & \mathbf{E}\left(1-2 e^{-y}+e^{-2 y}\right) \\
= & 1-2 \mathbf{E}\left(e^{-y}\right)+\mathbf{E}\left(e^{-2 y}\right) \\
= & 1 \\
\mathcal{I}_{12} & \mathbf{E}\left\{L_{1}(Y) L_{2}(Y)\right\} \\
= & \int_{-\infty}^{\infty}\left(1-e^{-y}\right)\left(-1+y-y e^{-y}\right) e^{-y-e^{-y}} d y \\
= & \int_{0}^{\infty}(1-x)(-1-\ln x+x \ln x) e^{-x} d x \\
& {\left[\operatorname{Let} e^{-y}=x \Longrightarrow-e^{-y} d y=d x\right]} \\
= & -\int_{0}^{\infty} e^{-x} d x+\int_{0}^{\infty} x e^{-x} d x-\int_{0}^{\infty}(\ln x) e^{-x} d x+2 \int_{0}^{\infty} x(\ln x) e^{-x} d x- \\
& \int_{0}^{\infty} x^{2}(\ln x) e^{-x} d x \\
& N_{0 w}^{\infty} \int_{0}^{\infty} e^{-x} d x=1, \quad \int_{0}^{\infty} x e^{-x} d x=1, \quad \int_{0}^{\infty}(\ln x) e^{-x} d x=-\gamma \\
= & -1+1+\gamma+2(1-\gamma)-(3-2 \gamma) \\
= & \gamma-1 \\
& \int_{0}^{\infty} x^{2}(\ln x) e^{-x} d x=3-2 \gamma \\
& \\
&
\end{array}
$$




$$
\begin{aligned}
\mathcal{I}_{22} & =E\left\{L_{2}^{2}(Y)\right\} \\
& =\int_{-\infty}^{\infty}\left(-1+y-y e^{-y}\right)^{2} e^{-y-e^{-y}} d y \\
& =-\int_{0}^{\infty}(-1-\ln x+x \ln x)^{2} \frac{x}{e^{x}}\left(-\frac{1}{x}\right) d x \\
& =\int_{0}^{\infty}(-1-\ln x+x \ln x)^{2} e^{-x} d x \\
& =\frac{\pi^{2}}{6}+(\gamma-1)^{2}
\end{aligned}
$$

where $\gamma$ is the Euler constant $(\gamma=0.57721566)$.

We construct an estimator $\left(T_{1 n}, T_{2 n}\right)$ of $(\theta, \sigma)$ defined by

$$
T_{i n}=n^{-1} \sum_{j=1}^{n} J_{i}\left(\frac{j}{n+1}\right) X_{j n}, \quad i=1,2
$$

where

$$
\left\{J_{1}(u), J_{2}(u)\right\}=\left\{L_{1}^{\prime}(y), L_{2}^{\prime}(y)\right\} \mathcal{I}^{-1}, \quad y=F^{-1}(u)
$$

and $\mathcal{I}^{-1}=\left(\left(\mathcal{I}^{i j}\right)\right)_{2 \times 2}$ is the inverse of the matrix $\mathcal{I}$.

$$
\text { We have, } \begin{aligned}
F(y) & =F\left(F^{-1}(u)\right)=u \\
\Longrightarrow e^{-e^{-y}} & =u \\
\Longrightarrow \ln u & =-e^{-y} \\
\Longrightarrow y & =-\ln (-\ln u) .
\end{aligned}
$$


To find value of $\left\{J_{1}(u), J_{2}(u)\right\}$ we have to find the values of $L_{1}^{\prime}(y), L_{2}^{\prime}(y)$ and $\mathcal{I}^{-1}$. Using equations $(2.6),(2.7),(2.8)$ and $\mathcal{I}_{12}=\mathcal{I}_{21}$ we have,

$$
\begin{aligned}
& \mathcal{I}=\left(\begin{array}{cc}
1 & (\gamma-1) \\
(\gamma-1) & \frac{\pi^{2}}{6}+(\gamma-1)^{2}
\end{array}\right) \\
& \mathcal{I}^{-1}=\frac{1}{\operatorname{det}(\mathcal{I})}\left(\begin{array}{cc}
1 & (\gamma-1) \\
(\gamma-1) & \frac{\pi^{2}}{6}+(\gamma-1)^{2}
\end{array}\right) \\
& \operatorname{det}(\mathcal{I})=\frac{\pi^{2}}{6}+(\gamma-1)^{2}-(\gamma-1)^{2}=\frac{\pi^{2}}{6} \\
& \mathcal{I}^{-1}=\frac{6}{\pi^{2}}\left(\begin{array}{cc}
\frac{\pi^{2}}{6}+(\gamma-1)^{2} & -(\gamma-1) \\
-(\gamma-1) & 1
\end{array}\right) \\
& L_{1}^{\prime}(y)=e^{-y} \\
& L_{2}^{\prime}(y)=1-e^{-y}+y e^{-y} .
\end{aligned}
$$

Using values of $L_{1}^{\prime}(y), L_{2}^{\prime}(y)$ and $\mathcal{I}^{-1}$ in (2.9) we get,

$$
\begin{aligned}
\left\{J_{1}(u), J_{2}(u)\right\} & =\left\{L_{1}^{\prime}(y), L_{2}^{\prime}(y)\right\} \mathcal{I}^{-1} \\
& =\left[e^{\ln (-\ln u)}, 1-e^{\ln (-\ln u)}-\ln (-\ln u) e^{\ln (-\ln u}\right] \mathcal{I}^{-1} \\
& =[-\ln u, 1+\ln u+\ln (-\ln u) \ln u] \frac{6}{\pi^{2}}\left(\begin{array}{cc}
\frac{\pi^{2}}{6}+(\gamma-1)^{2} & -(\gamma-1)) \\
-(\gamma-1) & 1
\end{array}\right) \\
& \left.=\frac{6}{\pi^{2}}\left(-\ln u\left(\frac{\pi^{2}}{6}+(\gamma-1)^{2}\right)-(\gamma-1)(1+\ln u+\ln (-\ln u) \ln u) \quad \ln u(\gamma-1)+1+\ln u+\ln (-\ln u) \ln u\right)\right) .
\end{aligned}
$$




$$
\begin{aligned}
J_{1}(u) & =\frac{6}{\pi^{2}}\left(-\ln u\left(\frac{\pi^{2}}{6}+(\gamma-1)^{2}\right)-\frac{6}{\pi^{2}}(\gamma-1)(1+\ln u+\ln (-\ln u) \ln u)\right. \\
& =-\ln u-\ln u \frac{6(\gamma-1)^{2}}{\pi^{2}}-\frac{6(\gamma-1)}{\pi^{2}}[1+\ln u(1+\ln (-\ln u))] \\
& =\frac{6(1-\gamma)}{\pi^{2}}+\ln u\left[\frac{6(1-\gamma)}{\pi^{2}}(1+\ln (-\ln u))-\frac{6(\gamma-1)^{2}}{\pi^{2}}-1\right] \\
& =\frac{6(1-\gamma)}{\pi^{2}}+\ln u\left[\frac{6(1-\gamma)}{\pi^{2}}[(1+\ln (-\ln u))-1+\gamma]-1\right] \\
& =\frac{6(1-\gamma)}{\pi^{2}}+\ln u\left[\frac{6(1-\gamma)}{\pi^{2}}[\ln (-\ln u)+\gamma]-1\right], 0<u<1 \\
J_{2}(u) & =\frac{6}{\pi^{2}}[\ln u(\gamma-1)+1+\ln u+\ln (-\ln u) \ln u] \\
& =\frac{6}{\pi^{2}}[\gamma \ln u-\ln u+1+\ln u+\ln (-\ln u) \ln u] \\
& =\frac{6}{\pi^{2}}[1+\ln u[\gamma+\ln (-\ln u)]], \quad 0<u<1 .
\end{aligned}
$$

It follows that $T_{1 n}$ and $T_{2 n}$ are asymptotically normally distributed with means $\theta$ and $\sigma$ and variances $\left(\sigma^{2} / n\right) \mathcal{I}^{11}$ and $\left(\sigma^{2} / n\right) \mathcal{I}^{22}$ respectively. This result follows as a special case of a theorem proved by Chernoff et al. (1967). Chernoff et al. (1967) used the technique of representing the ordered statistics in terms of independent and identically distributed random variables from a distribution $F$ to obtain the asymptotic distribution of linear combinations of functions of order statistics. Their main results have been reproduced by David. and Nagaraja (2003) in Theorem 11.4 (page 333) which is given in the following. 
Theorem 2.1. Assume that $V\left(X_{1}\right)<\infty$ and $J_{1}(u)$ is bounded and continuous. Then

$$
\lim _{n \rightarrow \infty} n E\left(T_{1 n}\right)=\mu\left(J_{1}, F\right), \lim _{n \rightarrow \infty} n V\left(T_{1 n}\right)=\sigma^{2}\left(J_{1}, F\right)
$$

where

$\mu\left(J_{1}, F\right)=\int_{0}^{1} J_{1}(u) F^{-1}(u) d u, \sigma^{2}\left(J_{1}, F\right)=2 \iint_{0<u<v<1} J_{1}(u) J_{1}(v) u(1-v) d F^{-1}(u) d F^{-1}(v)$,

and if $\sigma^{2}\left(J_{1}, F\right)<\infty$,

$$
\frac{T_{1 n}-E\left(T_{1 n}\right)}{\left[V\left(T_{1 n}\right)\right]^{1 / 2}} \stackrel{d}{\longrightarrow} N(0,1)
$$

Similar results hold for $T_{2 n}$.

Now the efficiency of our estimators can be justified using the following argument. If $V_{1}$ is the variance of an unbiased estimator of $\theta$ and $V_{2}$ is the variance of an unbiased estimator of $\sigma$, then $V_{1} \geq\left(\sigma^{2} / n\right) \mathcal{I}^{11}$ and $V_{2} \geq\left(\sigma^{2} / n\right) \mathcal{I}^{22}$ (Rao (1973), p.327). When the lower bound is attained, the estimator is defined to be an efficient estimator ( Rao (1973), p.346). This establishes the efficiency of the estimators.

Example 1. A simulated sample of size 50 is drawn from the type I (maximum) extreme value distribution with parameters $\theta=5$ and $\sigma=2$. The ordered sample observations (with four decimal places) are given below:

1.8338, 3.0794, 3.1010, 3.1049, 3.4371, 3.4964, 3.5632, 3.8717, 4.0057, 4.1325, 4.1501, 4.2857, 4.3513, 4.3907, 4.5819, 4.6228, 4.7619, 4.8774, 4.9583, 5.0723, 5.1336, 5.2534, 5.3320, 5.4117, 5.6466, 5.6922, 5.7550, 5.7862, 5.7948, 5.7957, 5.7970, 6.0634, 6.1819, 6.3656, 6.7001, 6.9049, 7.1456, 7.1730, 7.2507, 7.3662, 7.4530, 7.6647, 7.8524, 8.1934, 8.8646, 9.0670, 9.9792, 12.1116, 12.2717, 16.1719 
Using the above data, the estimates of $\theta$ and $\sigma$ are given by $T_{1 n}=4.9482$ and $T_{2 n}=1.9998$ respectively. 


\section{Chapter 3}

\section{Estimation of the parameters of the extreme value distribution on the basis of a censored sample}

Type I and type II censoring are most commonly used in the literature. A type I censoring mechanism is applied when each individual has a fixed potential censoring time $C_{i}>0$ such that $T_{i}$ is observed if $T_{i} \leq C_{i}$; otherwise we only know that $T_{i}>C_{i}$. Type I censoring often arises when a study is conducted over a specific time period. Let $T_{i}$ be the lifetime variable for a patient representing the duration of their remission measured from time of entry to the study and $C_{i}$ be the time between their date of entry and the end of study. For Type I censoring we have, $t_{i}=$ $\min \left(T_{i}, C_{i}\right), \quad \delta_{i}=I\left(T_{i} \leq C_{i}\right)$ (Lawless (2003)). The likelihood function for a type I censored sample is based on the probability distribution of $\left(t_{i}, \delta_{i}\right), i=1,2, \ldots \ldots, n$. Both $t_{i}$ and $\delta_{i}$ are random variables and their joint p.d.f. is

$$
f\left(t_{i}\right)^{\delta_{i}} \operatorname{Pr}\left(T_{i}>C_{i}\right)^{1-\delta_{i}}
$$


Type II censoring occurs when only the $r$ smallest lifetimes $t_{(1)} \leq \ldots \leq t_{(r)}$ in a random sample of $n$ are observed where $r$ is a specific integer between 1 and $n$ (Miller (1981)). This censoring arises when $n$ individuals start the study at the same time and the study terminates when $r$ failures (or lifetimes) have been observed. In this chapter we develop inference based on Type II censoring for extreme value distribution.

In this chapter we treat the problem of estimating $(\theta, \sigma)$ in the case of type II censoring. We obtain simple analytic expressions for the elements of the Fisher information matrix for this problem and then construct an estimator $\left(T_{1 n}^{*}, T_{2 n}^{*}\right)$ where additional weights are given to certain sample percentiles. Here again, the estimator $\left(T_{1 n}^{*}, T_{2 n}^{*}\right)$ is asymptotically normally distributed with mean vector $(\theta, \sigma)$ and covariance matrix $\sigma^{2}\left(n \mathcal{I}^{*}\right)^{-1}$.

In order to avoid trivial complications we assume in the sequel that for any $p$ $(0<p<1) n p$ is not an integer and define the sample percentile of order $p$ (also called the sample $p$ percentile) as the $[n p]+1$-th order statistic $X_{[n p]+1, n}$ where $[n p]$ is the largest integer less than or equal to $n p$. We study the case of type II censoring where the observations which lie in the sample percentile ranges $\left(0, p_{1}\right)$ and $\left(1-p_{2}, 1\right)$, are censored (i.e., unavailable), where $0<p_{1}<1-p_{2}<1$. Thus for each $i$, the observations lying between the sample $p_{1}$-percentile and the sample $\left(1-p_{2}\right)$-percentile (inclusive) are available. It will be convenient if we introduce the notations $q_{1}, q_{2}, r_{1}, r_{2}, \lambda_{1}, \lambda_{2}$ where $q_{1}=\ln p_{1}, q_{2}=\ln \left(1-p_{2}\right), r_{1}=\ln \left(-q_{1}\right)$, $r_{2}=\ln \left(-q_{2}\right), \lambda_{1}=-r_{1}$ and $\lambda_{2}=-r_{2}$.

We need to compute the asymptotic variance-covariance matrix which is related 
to the Fisher Information Matrix.

Theorem 3.1 The Fisher information matrix for the above problem is $\sigma^{-2} \mathcal{I}^{*}$, where $\mathcal{I}^{*}=\left(\left(\mathcal{I}_{i j}^{*}\right)\right)_{2 \times 2}$,

$$
\begin{aligned}
\mathcal{I}_{11}^{*}= & 1-p_{1}-p_{2}+\left(1-p_{2}\right) q_{2}^{2} / p_{2}, \\
\mathcal{I}_{12}^{*}= & p_{1}+p_{2}-1+p_{1} r_{1}-\left(1-p_{2}\right) r_{2}\left(1+q_{2}^{2} / p_{2}\right)+E_{1}\left(-q_{1}\right)-E_{1}\left(-q_{2}\right), \quad\left[\mathcal{I}_{12}^{*}=\mathcal{I}_{21}^{*}\right] \\
\mathcal{I}_{22}^{*}= & 1-p_{1}-p_{2}-p_{1} r_{1}\left(2+r_{1}\right)+\left(1-p_{2}\right) r_{2}\left(2+r_{2}+q_{2}^{2} r_{2} / p_{2}\right)+\left(r_{2}-r_{1}\right)\left(2 \gamma+r_{1}+r_{2}\right) \\
& +2\left[\left(1+r_{2}\right) E_{1}\left(-q_{2}\right)-\left(1+r_{1}\right) E_{1}\left(-q_{1}\right)\right]+2\left[S\left(1-p_{2}\right)-S\left(p_{1}\right)\right] .
\end{aligned}
$$

We derive the expressions for $\mathcal{I}_{11}^{*}, \mathcal{I}_{12}^{*}$ and $\mathcal{I}_{22}^{*}$ using the following.

$$
\begin{aligned}
& \mathcal{I}_{11}^{*}=\int_{\lambda_{1}}^{\lambda_{2}} L_{1}^{2}(y) f(y) d y+\frac{f^{2}\left(\lambda_{1}\right)}{p_{1}}+\frac{f^{2}\left(\lambda_{2}\right)}{p_{2}} \\
& \mathcal{I}_{12}^{*}=\int_{\lambda_{1}}^{\lambda_{2}} L_{1}(y) L_{2}(y) f(y) d y+\frac{\lambda_{1} f^{2}\left(\lambda_{1}\right)}{p_{1}}+\frac{\lambda_{2} f^{2}\left(\lambda_{2}\right)}{p_{2}} \\
& \mathcal{I}_{22}^{*}=\int_{\lambda_{1}}^{\lambda_{2}} L_{2}^{2}(y) f(y) d y+\frac{\lambda_{1}^{2} f^{2}\left(\lambda_{1}\right)}{p_{1}}+\frac{\lambda_{2}^{2} f^{2}\left(\lambda_{2}\right)}{p_{2}}
\end{aligned}
$$

(see Mandal and Samanta (2007) and Mandal and Samanta (2006)). 
Now, $f\left(\lambda_{1}\right)=e^{-\lambda_{1}} e^{-e^{-\lambda_{1}}}$

$$
\begin{aligned}
& =e^{\ln \left(-q_{1}\right)} e^{-e^{\ln \left(-q_{1}\right)}}\left[\text { Let, } \lambda_{1}=-r_{1}=-\ln \left(-q_{1}\right) \text { and } q_{1}=\ln p_{1}\right] \\
& =-q_{1} e^{q_{1}} \\
f\left(\lambda_{2}\right) & =e^{-\lambda_{2}} e^{-e^{-\lambda_{2}}} \\
& =e^{\ln \left(-q_{2}\right)} e^{-e^{\ln \left(-q_{2}\right)}}\left[\text { Let, } \lambda_{2}=-r_{2}=-\ln \left(-q_{2}\right) \text { and } q_{2}=\ln \left(1-p_{2}\right)\right] \\
& =-q_{2} e^{q_{2}} .
\end{aligned}
$$

Using values of $L_{1}(y), f(y), f\left(\lambda_{1}\right)$ and $f\left(\lambda_{2}\right)$ in equation (3.1), we ontain

$$
\begin{aligned}
\mathcal{I}_{11}^{*}= & \int_{\lambda_{1}}^{\lambda_{2}}\left(1-e^{-y}\right)^{2} e^{-y-e^{-y}} d y+p_{1} q_{1}^{2}+\left(1-p_{2}\right)^{2} q_{2}^{2} / p_{2} \\
= & \int_{-q_{2}}^{-q_{1}}(1-u)^{2} e^{-u} d u+p_{1} q_{1}^{2}+\left(1-p_{2}\right)^{2} q_{2}^{2} / p_{2} \\
& {\left[\text { Let }, u=e^{-y} \Longrightarrow d u=-e^{-y} d y\right] } \\
= & 1-p_{1}-p_{2}+\left(1-p_{2}\right) q_{2}^{2} / p_{2} .
\end{aligned}
$$

It can be verified that

$$
\int_{\lambda_{1}}^{\lambda_{2}} L_{1}(y) L_{2}(y) f(y) d y=-\int_{\lambda_{1}}^{\lambda_{2}} L_{1}(y) f(y) d y+\int_{\lambda_{1}}^{\lambda_{2}} y L_{1}^{\prime}(y) f(y) d y+\int_{\lambda_{1}}^{\lambda_{2}} y f^{\prime \prime}(y) d y
$$

Proof of equation (3.4) is given below 


$$
\begin{aligned}
& \int_{\lambda_{1}}^{\lambda_{2}} L_{1}(y) L_{2}(y) f(y) d y \\
& =\int_{\lambda_{1}}^{\lambda_{2}} L_{1}(y)\left(-1+y-y e^{-y}\right) f(y) d y \\
& =-\int_{\lambda_{1}}^{\lambda_{2}} L_{1}(y) f(y) d y+\int_{\lambda_{1}}^{\lambda_{2}} y f(y) L_{1}(y) d y-\int_{\lambda_{1}}^{\lambda_{2}} y e^{-y} f(y) L_{1}(y) d y \\
& =-\int_{\lambda_{1}}^{\lambda_{2}} L_{1}(y) f(y) d y+\int_{\lambda_{1}}^{\lambda_{2}} y f(y) L_{1}(y) d y-\int_{\lambda_{1}}^{\lambda_{2}} y f(y) e^{-y}\left(1-e^{-y}\right) d y \\
& =-\int_{\lambda_{1}}^{\lambda_{2}} L_{1}(y) f(y) d y+\int_{\lambda_{1}}^{\lambda_{2}} y f(y) L_{1}(y) d y-\int_{\lambda_{1}}^{\lambda_{2}} y e^{-y} f(y) d y \\
& +\int_{\lambda_{1}}^{\lambda_{2}} y e^{-2 y} f(y) d y \\
& =-\int_{\lambda_{1}}^{\lambda_{2}} L_{1}(y) f(y) d y+\int_{\lambda_{1}}^{\lambda_{2}} y f(y)\left(1-e^{-y}\right) d y-\int_{\lambda_{1}}^{\lambda_{2}} y e^{-y} f(y) d y \\
& +\int_{\lambda_{1}}^{\lambda_{2}} y e^{-2 y} f(y) d y \\
& =-\int_{\lambda_{1}}^{\lambda_{2}} L_{1}(y) f(y) d y+\int_{\lambda_{1}}^{\lambda_{2}} y e^{-3 y} e^{-e^{-y}} d y-\int_{\lambda_{1}}^{\lambda_{2}} y e^{-y} f(y) d y \\
& -\int_{\lambda_{1}}^{\lambda_{2}} y e^{-y} f(y) d y++\int_{\lambda_{1}}^{\lambda_{2}} y f(y) d y
\end{aligned}
$$




$$
\begin{aligned}
= & -\int_{\lambda_{1}}^{\lambda_{2}} L_{1}(y) f(y) d y+\int_{\lambda_{1}}^{\lambda_{2}} y e^{-3 y} e^{-e^{-y}} d y-2 \int_{\lambda_{1}}^{\lambda_{2}} y e^{-y} f(y) d y \\
& +\int_{\lambda_{1}}^{\lambda_{2}} y e^{-y} e^{-e^{-y}} d y \\
= & -\int_{\lambda_{1}}^{\lambda_{2}} L_{1}(y) f(y) d y+\int_{\lambda_{1}}^{\lambda_{2}} y e^{-y} f(y) d y+\int_{\lambda_{1}}^{\lambda_{2}} y e^{-3 y} e^{-e^{-y}} d y \\
& -2 \int_{\lambda_{1}}^{\lambda_{2}} y e^{-2 y} e^{-e^{-y}} d y-\int_{\lambda_{1}}^{\lambda_{2}} y e^{-2 y} e^{-e^{-y}} d y+\int_{\lambda_{1}}^{\lambda_{2}} y e^{-y} e^{-e^{-y}} d y \\
= & -\int_{\lambda_{1}}^{\lambda_{2}} L_{1}(y) f(y) d y+\int_{\lambda_{1}}^{\lambda_{2}} y L_{1}^{\prime}(y) f(y) d y+\int_{\lambda_{1}}^{\lambda_{2}} y f^{\prime \prime}(y) d y \\
& {[\text { Proved }] }
\end{aligned}
$$

We now derive each part of equation (3.5).

First part of equation (3.5) :

$$
\begin{aligned}
\int_{\lambda_{1}}^{\lambda_{2}}-L_{1}(y) f(y) d y & =\int_{\lambda_{1}}^{\lambda_{2}}\left(-1+e^{-y}\right) e^{-y} e^{-e^{-y}} d y \\
& =\int_{-q_{2}}^{-q_{1}}(-1+u) e^{-u} d u \quad\left[\text { let, } e^{-y}=u \Longrightarrow-e^{-y} d y=d u\right] \\
& =-\int_{-q_{2}}^{-q_{1}} e^{-u} d u+\int_{-q_{2}}^{-q_{1}} u e^{-u} d u \\
& =\left\{e^{-u}\right\}_{u=-q_{2}}^{u=-q_{1}}+\left\{-u e^{-u}\right\}_{u=-q_{2}}^{u=-q_{1}}+\int_{-q_{2}}^{-q_{1}} e^{-u} d u
\end{aligned}
$$




$$
\begin{aligned}
& =e^{q_{1}}-e^{q_{2}}+q_{1} e^{q_{1}}-q_{2} e^{q_{2}}-\left\{e^{-u}\right\}_{u=-q_{2}}^{u=-q_{1}} \\
& =e^{q_{1}}-e^{q_{2}}+q_{1} e^{q_{1}}-q_{2} e^{q_{2}}-e^{q_{1}}+e^{q_{2}} \\
& =q_{1} e^{q_{1}}-q_{2} e^{q_{2}} \\
& =q_{1} p_{1}-q_{2}\left(1-p_{2}\right) .
\end{aligned}
$$

Second part of equation (3.5):

$$
\begin{aligned}
\int_{\lambda_{1}}^{\lambda_{2}} y L_{1}^{\prime}(y) f(y) d y & =\int_{\lambda_{1}}^{\lambda_{2}} y e^{-y} \exp \left(-y-e^{-y}\right) d y \\
& =\int_{\lambda_{1}}^{\lambda_{2}} y e^{-y} e^{-y} e^{-e^{-y}} d y \quad\left[\text { let, } e^{-y}=u\right] \\
& =\int_{-q_{1}}^{-q_{2}} u(\ln u) e^{-u} d u \\
& =\left\{u(\ln u) e^{-u}\right\}_{-q_{2}}^{-q_{1}}+\int_{-q_{1}}^{-q_{2}} e^{-u}(1+\ln u) d u \\
& =\left\{u(\ln u) e^{-u}\right\}_{-q_{2}}^{-q_{1}}+\int_{-q_{1}}^{-q_{2}} e^{-u} d u+\int_{-q_{1}}^{-q_{2}} e^{-u}(\ln u) d u \\
& =\left\{u(\ln u) e^{-u}\right\}_{-q_{2}}^{-q_{1}}+\left\{e^{-u}\right\}_{-q_{2}}^{-q_{1}}+\int_{-q_{1}}^{-q_{2}} e^{-u}(\ln u) d u
\end{aligned}
$$




$$
\begin{aligned}
= & \left\{u(\ln u) e^{-u}\right\}_{-q_{2}}^{-q_{1}}+\left\{e^{-u}\right\}_{-q_{2}}^{-q_{1}}+\left\{(\ln u) e^{-u}\right\}_{-q_{2}}^{-q_{1}}+\int_{-q_{1}}^{-q_{2}} e^{-u} \frac{1}{u} d u \\
= & -q_{1} \ln \left(-q_{1}\right) e^{q_{1}}+q_{2} \ln \left(-q_{2}\right) e^{q_{2}}+e^{q_{1}}-e^{q_{2}}-\ln \left(-q_{2}\right) e^{q_{2}} \\
& +\ln \left(-q_{1}\right) e^{q_{1}}+\int_{-q_{1}}^{-q_{2}} e^{-u} \frac{1}{u} d u \\
= & -q_{1} r_{1} p_{1}+q_{2} r_{2}\left(1-p_{2}\right)+p_{1}-1+p_{2}-r_{2}\left(1-p_{2}\right)+r_{1} p_{1} \\
& +\int_{-q_{1}}^{-q_{2}} e^{-u} \frac{1}{u} d u \\
= & p_{1}+p_{2}-1+p_{1} r_{1}\left(1-q_{1}\right)+\left(1-p_{2}\right) q_{2} r_{2}-r_{2}\left(1-p_{2}\right)+\int_{-q_{1}}^{-q_{2}} e^{-u} \frac{1}{u} d u \\
= & p_{1}+p_{2}-1+p_{1} r_{1}\left(1-q_{1}\right)-\left(1-p_{2}\right) r_{2}\left(1-q_{2}\right)+E_{1}\left(-q_{1}\right)-E_{1}\left(-q_{2}\right) \\
= & p_{1}+p_{2}-1+p_{1}\left(1-q_{1}\right) r_{1}-\left(1-p_{2}\right)\left(1-q_{2}\right) r_{2}+E_{1}\left(-q_{1}\right)-E_{1}\left(-q_{2}\right)
\end{aligned}
$$

Third part of equation (3.5):

$$
\begin{aligned}
\int_{\lambda_{1}}^{\lambda_{2}} y f^{\prime \prime}(y) d y & \\
= & y\left(e^{-3 y} e^{-e^{-y}}-3 e^{-2 y} e^{-e^{-y}}+e^{-y} e^{-e^{-y}}\right) d y \\
= & \left.\int_{\lambda_{1}}^{\lambda_{2}} y e^{-3 y} e^{-e^{-y}} d y-3 \int_{\lambda_{1}}^{\lambda_{2}} y e^{-2 y} e^{-e^{-y}} d y+\int_{\lambda_{1}}^{\lambda_{2}} y e^{-y} e^{-e^{-y}}\right) d y \\
& {\left[\text { Let, } e^{-y}=u \Longrightarrow-e^{-y} d y=d u\right] }
\end{aligned}
$$




$$
\begin{aligned}
= & \int_{-q_{1}}^{-q_{2}} u^{2}(\ln u) e^{-u} d u-3 \int_{-q_{1}}^{-q_{2}} u(\ln u) e^{-u} d u+\int_{-q_{1}}^{-q_{2}}(\ln u) e^{-u} d u \\
= & \left\{u^{2}(\ln u) e^{-u}\right\}_{-q_{2}}^{-q_{1}}+\left\{u e^{-u}\right\}_{-q_{2}}^{-q_{1}}+\left\{e^{-u}\right\}_{-q_{2}}^{-q_{1}}+2 \int_{-q_{1}}^{-q_{2}} u(\ln u) e^{-u} d u \\
& -3 \int_{-q_{1}}^{-q_{2}} u(\ln u) e^{-u} d u+\int_{-q_{1}}^{-q_{2}}(\ln u) e^{-u} d u
\end{aligned}
$$

[using equation (3.9)]

$$
\begin{aligned}
= & \left\{u^{2}(\ln u) e^{-u}\right\}_{-q_{2}}^{-q_{1}}+\left\{u e^{-u}\right\}_{-q_{2}}^{-q_{1}}+\left\{e^{-u}\right\}_{-q_{2}}^{-q_{1}}-\int_{-q_{1}}^{-q_{2}} u(\ln u) e^{-u} d u \\
& +\int_{-q_{1}}^{-q_{2}}(\ln u) e^{-u} d u \\
= & \left\{u^{2}(\ln u) e^{-u}\right\}_{-q_{2}}^{-q_{1}}+\left\{u e^{-u}\right\}_{-q_{2}}^{-q_{1}}+\left\{e^{-u}\right\}_{-q_{2}}^{-q_{1}}-\left\{u(\ln u) e^{-u}\right\}_{-q_{2}}^{-q_{1}}-\left\{e^{-u}\right\}_{-q_{2}}^{-q_{1}} \\
& -\int_{-q_{1}}^{-q_{2}} e^{-u}(\ln u) d u+\int_{-q_{1}}^{-q_{2}}(\ln u) e^{-u} d u \\
= & \left\{u^{2}(\ln u) e^{-u}\right\}_{-q_{2}}^{-q_{1}}+\left\{u e^{-u}\right\}_{-q_{2}}^{-q_{1}}+\left\{e^{-u}\right\}_{-q_{2}}^{-q_{1}}-\left\{u(\ln u) e^{-u}\right\}_{-q_{2}}^{-q_{1}}-\left\{e^{-u}\right\}_{-q_{2}}^{-q_{1}} \\
= & q_{1}^{2} \ln \left(-q_{1}\right) e^{q_{1}}-q_{2}^{2} \ln \left(-q_{2}\right) e^{q_{2}}-q_{1} e^{q_{1}}+q_{2} e^{q_{2}}+e^{q_{1}}-e^{q_{2}} \\
& +q_{1} \ln \left(-q_{1}\right) e^{q_{1}}-q_{2} \ln \left(-q_{2}\right) e^{q_{2}}-e^{q_{1}}+e^{q_{2}} \\
= & q_{1}^{2} r_{1} e^{q_{1}}-q_{2}^{2} r_{2} e^{q_{2}}-q_{1} e^{q_{1}}+q_{2} e^{q_{2}}+e^{q_{1}}-e^{q_{2}}+q_{1} r_{1} e^{q_{1}}-q_{2} r_{2} e^{q_{2}}-e^{q_{1}}+e^{q_{2}} \\
= & q_{1}^{2} r_{1} p_{1}-q_{2}^{2} r_{2}\left(1-p_{2}\right)-q_{1} p_{1}+q_{2}\left(1-p_{2}\right)+q_{1} r_{1} p_{1}-q_{2} r_{2}\left(1-p_{2}\right) \\
& {\left[r_{1}=\ln \left(-q_{1}\right), r_{2}=\ln \left(-q_{2}\right), q_{1}=\ln p_{1} \text { and } q_{2}=\ln \left(1-p_{2}\right)\right] }
\end{aligned}
$$




$$
\begin{aligned}
& =q_{1}^{2} r_{1} p_{1}-q_{1} p_{1}+q_{1} r_{1} p_{1}-q_{2}^{2} r_{2}\left(1-p_{2}\right)+q_{2}\left(1-p_{2}\right)-q_{2} r_{2}\left(1-p_{2}\right) \\
& =q_{1} p_{1}\left(-1+q_{1} r_{1}+r_{1}\right)-\left(1-p_{2}\right)\left(q_{2}^{2} r_{2}-q_{2}+q_{2} r_{2}\right) \\
& =q_{1} p_{1}\left[r_{1}\left(1+q_{1}\right)-1\right]-\left(1-p_{2}\right) q_{2}\left[r_{2}\left(1+q_{2}\right)-1\right]
\end{aligned}
$$

We prove the expression of $\int_{-q_{1}}^{-q_{2}} u^{2}(\ln u) e^{-u} d u$ in the following :

$$
\begin{aligned}
\int_{-q_{1}}^{-q_{2}} & u^{2}(\ln u) e^{-u} d u \\
& =\left\{-u^{2}(\ln u) e^{-u}\right\}_{-q_{1}}^{-q_{2}}+\int_{-q_{1}}^{-q_{2}} e^{-u}(u+2 u \ln u) d u
\end{aligned}
$$

[using integration by parts formula where $U=u^{2}(\ln u), d V=e^{-u} d u$ ]

$=\left\{u^{2}(\ln u) e^{-u}\right\}_{-q_{2}}^{-q_{1}}+\int_{-q_{1}}^{-q_{2}} u e^{-u} d u+2 \int_{-q_{1}}^{-q_{2}} u(\ln u) e^{-u} d u$

$=\left\{u^{2}(\ln u) e^{-u}\right\}_{-q_{2}}^{-q_{1}}-\left\{u e^{-u}\right\}_{-q_{1}}^{-q_{2}}+\int_{-q_{1}}^{-q_{2}} e^{-u} d u+2 \int_{-q_{1}}^{-q_{2}} u(\ln u) e^{-u} d u$

$=\left\{u^{2}(\ln u) e^{-u}\right\}_{-q_{2}}^{-q_{1}}+\left\{u e^{-u}\right\}_{-q_{2}}^{-q_{1}}+\left\{e^{-u}\right\}_{-q_{2}}^{-q_{1}}$

$+2 \int_{-q_{1}}^{-q_{2}} u(\ln u) e^{-u} d u$ 
Now,

$$
\begin{aligned}
\frac{\lambda_{1} f^{2}\left(\lambda_{1}\right)}{p_{1}}+\frac{\lambda_{2} f^{2}\left(\lambda_{2}\right)}{p_{2}} & =\frac{\lambda_{1} q_{1}^{2} e^{2 q_{1}}}{p_{1}}+\frac{\lambda_{2} q_{2}^{2} e^{2 q_{2}}}{p_{2}} \\
& =-p_{1} q_{1}^{2} r_{1}-\frac{\left(1-p_{2}\right)^{2} q_{2}^{2} r_{2}}{p_{2}} \\
& =-\left\{p_{1} q_{1}^{2} r_{1}+\frac{\left(1-p_{2}\right)^{2} q_{2}^{2} r_{2}}{p_{2}}\right\} .
\end{aligned}
$$

Using the equations (3.6), (3.7),(3.8) and (3.10) we obtain

$$
\begin{aligned}
\mathcal{I}_{12}^{*}= & \int_{\lambda_{1}}^{\lambda_{2}} L_{1}(y) L_{2}(y) f(y) d y+\frac{\lambda_{1} f^{2}\left(\lambda_{1}\right)}{p_{1}}+\frac{\lambda_{2} f^{2}\left(\lambda_{2}\right)}{p_{2}} \\
= & p_{1} q_{1}-\left(1-p_{2}\right) q_{2}+p_{1}+p_{2}-1+p_{1}\left(1-q_{1}\right) r_{1}-\left(1-p_{2}\right)\left(1-q_{2}\right) r_{2}+E_{1}\left(-q_{1}\right) \\
& -E_{1}\left(-q_{2}\right)+p_{1} q_{1}\left[\left(1+q_{1}\right) r_{1}-1\right]-\left(1-p_{2}\right) q_{2}\left[\left(1+q_{2}\right) r_{2}-1\right]-p_{1} q_{1}^{2} r_{1} \\
& -\frac{\left(1-p_{2}\right)^{2} q_{2}^{2} r_{2}}{p_{2}} \\
= & p_{1}+p_{2}-1+p_{1} q_{1}-q_{2}+p_{2} q_{2}+p_{1} r_{1}-p_{1} q_{1} r_{1}+p_{1} q_{1}\left(r_{1}+r_{1} q_{1}-1\right) \\
& -\left(1-p_{2}\right)\left[r_{2}\left(1-q_{2}\right)+q_{2}\left(\left(1+q_{2}\right) r_{2}-1\right)\right]+E_{1}\left(-q_{1}\right)-E_{1}\left(-q_{2}\right)-p_{1} q_{1}^{2} r_{1} \\
& -\frac{\left(1-p_{2}\right)^{2} q_{2}^{2} r_{2}}{p_{2}} \\
= & p_{1}+p_{2}-1-q_{2}+p_{2} q_{2}+p_{1} r_{1}-p_{1} q_{1} r_{1}+p_{1} q_{1} r_{1}+p_{1} q_{1}^{2} r_{1} \\
& -\left(1-p_{2}\right)\left(r_{2}-r_{2} q_{2}+r_{2} q_{2}+r_{2} q_{2}^{2}-q_{2}\right)+E_{1}(-q)-E_{1}\left(-q_{2}\right)-p_{1} q_{1}^{2} r_{1} \\
& -\frac{\left(1-p_{2}\right)^{2} q_{2}^{2} r_{2}}{p_{2}} \\
& -(1-1)
\end{aligned}
$$




$$
\begin{aligned}
= & p_{1}+p_{2}-1-q_{2}+p_{2} q_{2}+p_{1} r_{1}-\left(1-p_{2}\right)\left(r_{2}+r_{2} q_{2}^{2}-q_{2}+\frac{\left(1-p_{2}\right) q_{2}^{2} r_{2}}{p_{2}}\right) \\
& +E_{1}\left(-q_{1}\right)-E_{1}\left(q_{2}\right) \\
= & p_{1}+p_{2}-1-q_{2}+p_{2} q_{2}+p_{1} r_{1}-\left(1-p_{2}\right) \frac{\left(r_{2} p_{2}+r_{2} p_{2} q_{2}^{2}-p_{2} q_{2}+q_{2}^{2} r_{2}-p_{2} q_{2}^{2} r_{2}\right)}{p_{2}} \\
& +E_{1}\left(-q_{1}\right)-E_{1}\left(q_{2}\right) \\
& p_{1}+p_{2}-1-q_{2}+p_{2} q_{2}+p_{1} r_{1}-\left(1-p_{2}\right) \frac{\left(r_{2} p_{2}-p_{2} q_{2}+q_{2}^{2} r_{2}\right)}{p_{2}}+E_{1}\left(-q_{1}\right)-E_{1}\left(q_{2}\right) \\
= & p_{1}+p_{2}-1+p_{1} r_{1}-q_{2}\left(1-p_{2}\right)-\left(1-p_{2}\right) \frac{\left(r_{2} p_{2}-p_{2} q_{2}+q_{2}^{2} r_{2}\right)}{p_{2}}+E_{1}\left(-q_{1}\right)-E_{1}\left(q_{2}\right) \\
= & p_{1}+p_{2}-1+p_{1} r_{1}-\left(1-p_{2}\right)\left[q_{2}+\frac{\left(r_{2} p_{2}-p_{2} q_{2}+q_{2}^{2} r_{2}\right)}{p_{2}}\right]+E_{1}\left(-q_{1}\right)-E_{1}\left(q_{2}\right) \\
= & p_{1}+p_{2}-1+p_{1} r_{1}-\left(1-p_{2}\right) r_{2}\left(1+q_{2}^{2} / p_{2}\right)+E_{1}\left(-q_{1}\right)-E_{1}\left(-q_{2}\right) .
\end{aligned}
$$

Next we will find the expression for $\mathcal{I}_{22}^{*}$ using following:

$$
\begin{aligned}
\mathcal{I}_{22}^{*} & =\int_{\lambda_{1}}^{\lambda_{2}} L_{2}^{2}(y) f(y) d y+\frac{\lambda_{1}^{2} f^{2}\left(\lambda_{1}\right)}{p_{1}}+\frac{\lambda_{2}^{2} f^{2}\left(\lambda_{2}\right)}{p_{2}}, \\
L_{2}^{2}(y) & =\left(-1+y-y e^{-y}\right)^{2} \\
& =1+y^{2}+y^{2} e^{-2 y}-2 y+2 y e^{-y}-2 y^{2} e^{-y} \\
& =1-2 y+2 y e^{-y}+y^{2}\left\{e^{-2 y}-2 e^{-y}+1\right\} \\
& =1-2 y+2 y e^{-y}+y^{2}\left\{e^{-2 y}-2 e^{-y}+1+e^{-y}-e^{-y}\right\}
\end{aligned}
$$




$$
\begin{aligned}
= & 1-2 y+2 y e^{-y}+y^{2}\left\{e^{-y}+\frac{e^{-3 y-e^{-y}}-3 e^{-2 y-e^{-y}}+e^{-y-e^{-y}}}{e^{-y-e^{-y}}}\right\} \\
= & 1-2 y\left(1-e^{-y}\right)+y^{2}\left\{e^{-y}+\frac{f^{\prime \prime}(y)}{e^{-y-e^{-y}}}\right\} \\
= & 1-2 y\left(1-e^{-y}\right)+y^{2}\left\{L_{1}^{\prime}(y)+\frac{f^{\prime \prime}(y)}{f(y)}\right\} \\
& \quad\left[\text { using equation }(2.3) \text { and } L_{1}^{\prime}(y)=e^{-y}\right] \\
= & 1-2 y L_{1}(y)+y^{2}\left\{L_{1}^{\prime}(y)+\frac{f^{\prime \prime}(y)}{f(y)}\right\} .
\end{aligned}
$$

Hence,

$$
\begin{aligned}
\int_{\lambda_{1}}^{\lambda_{2}} L_{2}^{2}(y) f(y) d y= & 1-p_{1}-p_{2}-2 \int_{\lambda_{1}}^{\lambda_{2}} y f(y) d y+2 \int_{\lambda_{1}}^{\lambda_{2}} y e^{-y} f(y) d y \\
& +\int_{\lambda_{1}}^{\lambda_{2}} y^{2} e^{-y} f(y) d y+\int_{\lambda_{1}}^{\lambda_{2}} y^{2} f^{\prime \prime}(y) d y .
\end{aligned}
$$

We prove this in the following.

$$
\begin{aligned}
L_{2}^{2}(y)= & 1-2 y L_{1}(y)+y^{2}\left\{L_{1}^{\prime}(y)+\frac{f^{\prime \prime}(y)}{f(y)}\right\} \\
\int_{\lambda_{1}}^{\lambda_{2}} L_{2}^{2}(y) f(y) d y= & \int_{\lambda_{1}}^{\lambda_{2}} f(y) d y-2 \int_{\lambda_{1}}^{\lambda_{2}} y L_{1}(y) f(y) d y+\int_{\lambda_{1}}^{\lambda_{2}} y^{2} L_{1}^{\prime}(y) f(y)+\int_{\lambda_{1}}^{\lambda_{2}} y^{2} f^{\prime \prime}(y) d y \\
= & 1-p_{2}-p_{1}-2 \int_{\lambda_{1}}^{\lambda_{2}} y\left(1-e^{-y}\right) f(y) d y+\int_{\lambda_{1}}^{\lambda_{2}} y^{2} e^{-y} f(y) d y \\
& +\int_{\lambda_{1}}^{\lambda_{2}} y^{2} f^{\prime \prime}(y) d y
\end{aligned}
$$




$$
\begin{aligned}
= & 1-p_{1}-p_{2}-2 \int_{\lambda_{1}}^{\lambda_{2}} y f(y) d y+2 \int_{\lambda_{1}}^{\lambda_{2}} y e^{-y} f(y) d y+\int_{\lambda_{1}}^{\lambda_{2}} y^{2} e^{-y} f(y) d y \\
& +\int_{\lambda_{1}}^{\lambda_{2}} y^{2} f^{\prime \prime}(y) d y . \quad \text { (Proved) }
\end{aligned}
$$

A straightforward calculation gives

$$
\begin{aligned}
\int_{\lambda_{1}}^{\lambda_{2}} y f(y) d y= & \int_{\lambda_{1}}^{\lambda_{2}} y \exp \left(-y-e^{-y}\right) d y \\
= & \int_{-q_{1}}^{-q_{2}}(\ln u) e^{-u} d u \\
& {[\text { using Integration by parts formula }] } \\
= & \left\{-\ln u e^{-u}\right\}_{-q_{1}}^{-q_{2}}+\int_{-q_{1}}^{-q_{2}} e^{-u} \frac{1}{u} d u \\
= & -\ln \left(-q_{2}\right) e^{q_{2}}+\ln \left(-q_{1}\right) e^{q_{1}}+E_{1}\left(-q_{1}\right)-E_{1}\left(-q_{2}\right) \\
= & p_{1} r_{1}-\left(1-p_{2}\right) r_{2}+E_{1}\left(-q_{1}\right)-E_{1}\left(-q_{2}\right)
\end{aligned}
$$

and

$$
\begin{aligned}
\int_{\lambda_{1}}^{\lambda_{2}} y^{2} f^{\prime \prime} & (y) d y \\
= & \int_{\lambda_{1}}^{\lambda_{2}} y^{2}\left(e^{-3 y} e^{-e^{-y}}-3 e^{-2 y} e^{-e^{-y}}+e^{-y} e^{-e^{-y}}\right) d y \\
= & \int_{\lambda_{1}}^{\lambda_{2}} y^{2} e^{-3 y} e^{-e^{-y}} d y-3 \int_{\lambda_{1}}^{\lambda_{2}} y^{2} e^{-2 y} e^{-e^{-y}} d y+\int_{\lambda_{1}}^{\lambda_{2}} y^{2} e^{-y} e^{-e^{-y}} d y \\
& \quad[\text { using equation (3.15), (3.17) and (3.18) }]
\end{aligned}
$$




$$
\begin{aligned}
= & -p_{1} q_{1}^{2} r_{1}^{2}+q_{2}^{2} r_{2}^{2}-p_{2} q_{2}^{2} r_{2}^{2}+2 p_{1} q_{1} r_{1}-2 q_{2} r_{2}+2 p_{2} q_{2} r_{2}+2\left(1-p_{1}-p_{2}\right) \\
& -2 p_{1} r_{1}+2\left(1-p_{2}\right) r_{2}+2 p_{1} q_{1} r_{1}^{2}-2\left(1-p_{2}\right) q_{2} r_{2}^{2}-4 p_{1} r_{1}+4\left(1-p_{2}\right) r_{2} \\
& -2 p_{1} r_{1}^{2}+2\left(1-p_{2}\right) r_{2}^{2}+6 \int_{-q_{2}}^{-q_{1}} \frac{e^{-u}}{u} d u+4 \int_{-q_{2}}^{-q_{1}} \frac{e^{-u} l n u}{u} d u-3 p_{1} q_{1} r_{1}^{2} \\
& +3 p_{1} r_{1}^{2}-3\left(1-p_{2}\right)\left(1-q_{2}\right) r_{2}^{2}+6\left[p_{1} r_{1}-\left(1-p_{2}\right) r_{2}+E_{1}\left(-q_{1}\right)-E_{1}\left(-q_{2}\right)\right] \\
& -6 \int_{-q_{2}}^{-q_{1}} \frac{e^{-u} \operatorname{lnu}}{u} d u-p_{1} r_{1}^{2}+\left(1-p_{2}\right) r_{2}^{2}+2 \int_{-q_{2}}^{-q_{1}} \frac{e^{-u} \operatorname{lnu}}{u} d u \\
= & 2\left(1-p_{1}-p_{2}\right)-p_{1} q_{1} r_{1}\left(r_{1}+q_{1} r_{1}-2\right) \\
& -2 p_{1} r_{1}-4 p_{1} r_{1}-2 p_{1} r_{1}^{2}+3 p_{1} r_{1}^{2}+6 p_{1} r_{1}-p_{1} r_{1}^{2} \\
& +\left(1-p_{2}\right)\left(q_{2}^{2} r_{2}^{2}-2 q_{2} r_{2}+2 r_{2}-2 q_{2} r_{2}^{2}+4 r_{2}+2 r_{2}^{2}-3 r_{2}^{2}+3 q_{2} r_{2}^{2}-6 r_{2}+r_{2}^{2}\right) \\
= & 2\left(1-p_{1}-p_{2}\right)-p_{1} q_{1} r_{1}\left\{\left(1+q_{1}\right) r_{1}-2\right\}+\left(1-p_{2}\right)\left(q_{2}^{2} r_{2}^{2}-2 q_{2} r_{2}+q_{2} r_{2}^{2}\right) \\
= & 2\left(1-p_{1}-p_{2}\right)-p_{1} q_{1} r_{1}\left\{\left(1+q_{1}\right) r_{1}-2\right\}+\left(1-p_{2}\right) q_{2} r_{2}\left\{\left(1+q_{2}\right) r_{2}-2\right\} .(3.14)
\end{aligned}
$$

$$
\begin{aligned}
\int_{\lambda_{1}}^{\lambda_{2}} & y^{2} e^{-3 y} e^{-e^{-y}} d y \\
& =\int_{-q_{2}}^{-q_{1}} u^{2} e^{-u}(\ln u)^{2} d u \\
& =\left\{-u^{2}(\ln u)^{2} e^{-u}\right\}_{-q_{2}}^{-q_{1}}+\int_{-q_{2}}^{-q_{1}} e^{-u}\left(2 u \ln u+2 u(\ln u)^{2}\right) d u
\end{aligned}
$$

[using itergration by parts formula where $U^{2}=u^{2}(\ln u)^{2}, \quad d V=e^{-u} d u$ ]

$$
=\left\{-u^{2}(\ln u)^{2} e^{-u}\right\}_{-q_{2}}^{-q_{1}}+2 \int_{-q_{2}}^{-q_{1}} e^{-u} u(\ln u) d u+2 \int_{-q_{2}}^{-q_{1}} e^{-u} u(\ln u)^{2} d u
$$




$$
\begin{aligned}
& =\left\{-u^{2}(\ln u)^{2} e^{-u}\right\}_{-q_{2}}^{-q_{1}}+2\left\{-u(\ln u) e^{-u}\right\}_{-q_{2}}^{-q_{1}}+2\left\{-e^{-u}\right\}_{-q_{2}}^{-q_{1}} \\
& +2\left\{-(\ln u) e^{-u}\right\}_{-q_{2}}^{-q_{1}}+2 \int_{-q_{2}}^{-q_{1}} \frac{e^{-u}}{u} d u+2\left\{-u(\ln u)^{2} e^{-u}\right\}_{-q_{2}}^{-q_{1}} \\
& +4\left\{-\ln u e^{-u}\right\}_{-q_{2}}^{-q_{1}}+4 \int_{-q_{2}}^{-q_{1}} \frac{e^{-u}}{u} d u+2\left\{-(\ln u)^{2} e^{-u}\right\}_{-q_{2}}^{-q_{1}} \\
& +4 \int_{-q_{2}}^{-q_{1}} \frac{e^{-u}(\ln u)}{u} d u \quad \text { [using equation (3.7) and (3.16)] } \\
& =-q_{1}^{2}\left[\ln \left(-q_{1}\right)\right]^{2} e^{q_{1}}+q_{2}^{2}\left[\ln \left(-q_{2}\right)\right]^{2} e^{q_{2}}+2\left[q_{1} \ln \left(-q_{1}\right) e^{q_{1}}-q_{2} \ln \left(-q_{2}\right) e^{q_{2}}\right] \\
& +2\left(-e^{q_{1}}+e^{q_{2}}\right)+6 \int_{-q_{2}}^{-q_{1}} \frac{e^{-u}}{u} d u+2\left[-\ln \left(-q_{1}\right) e_{1}^{q}+\ln \left(-q_{2}\right) e^{q_{2}}\right] \\
& +2\left[q_{1}\left(\ln \left(-q_{1}\right)\right)^{2} e^{q_{1}}-q_{2}\left(\ln \left(-q_{2}\right)\right)^{2} e^{q_{2}}\right]+4\left[-\ln \left(-q_{1}\right) e^{q_{1}}+\ln \left(-q_{2}\right) e^{q_{2}}\right] \\
& +2\left[-\left(\ln \left(-q_{1}\right)\right)^{2} e^{q_{1}}+\left(\ln \left(-q_{2}\right)\right)^{2} e^{q_{2}}+4 \int_{-q_{2}}^{-q_{1}} \frac{e^{-u} \ln u}{u} d u\right. \\
& =-p_{1} q_{1}^{2} r_{1}^{2}+\left(1-p_{2}\right) q_{2}^{2} r_{2}^{2}+2\left[p_{1} q_{1} r_{1}-\left(1-p_{2}\right) q_{2} r_{2}\right]+2\left(-p_{1}+1-p_{2}\right) \\
& +2\left[p_{1} r_{1}+\left(1-p_{2}\right) r_{2}\right]+2\left[p_{1} q_{1} r_{1}^{2}-\left(1-p_{2}\right) q_{2} r_{2}^{2}\right]+4\left[-p_{1} r_{1}+\left(1-p_{2}\right) r_{2}\right] \\
& +2\left[-p_{1} r_{1}^{2}+\left(1-p_{2}\right) r_{2}^{2}\right]+6 \int_{-q_{2}}^{-q_{1}} \frac{e^{-u}}{u} d u+4 \int_{-q_{2}}^{-q_{1}} \frac{e^{-u} \ln u}{u} d u \\
& =-p_{1} q_{1}^{2} r_{1}^{2}+q_{2}^{2} r_{2}^{2}-p_{2} q_{2}^{2} r_{2}^{2}+2 p_{1} q_{1} r_{1}-2 q_{2} r_{2}+2 p_{2} q_{2} r_{2}+2\left(1-p_{1}-p_{2}\right)-2 p_{1} r_{1} \\
& +2\left(1-p_{2}\right) r_{2}+2 p_{1} q_{1} r_{1}^{2}-2\left(1-p_{2}\right) q_{2} r_{2}^{2}-4 p_{1} r_{1}+4\left(1-p_{2}\right) r_{2}-2 p_{1} r_{1}^{2} \\
& +2\left(1-p_{2}\right) r_{2}^{2}+6 \int_{-q_{2}}^{-q_{1}} \frac{e^{-u}}{u} d u+4 \int_{-q_{2}}^{-q_{1}} \frac{e^{-u} \ln u}{u} d u \text {. }
\end{aligned}
$$


$\int_{-q_{2}}^{-q_{1}} e^{-u} u(\ln u)^{2} d u$

(using integration by parts formula where $U=u(\ln u)^{2}, d V=e^{-u}$ )

$$
\begin{aligned}
& =\left\{-u(\ln u)^{2} e^{-u}\right\}_{-q_{2}}^{-q_{1}}+\int_{-q_{2}}^{-q_{1}} e^{-u}\left(2 \ln u+(\ln u)^{2}\right) d u \\
& =\left\{-u(\ln u)^{2} e^{-u}\right\}_{-q_{2}}^{-q_{1}}+2 \int_{-q_{2}}^{-q_{1}} e^{-u}(\ln u) d u+\int_{-q_{2}}^{-q_{1}} e^{-u}(\ln u)^{2} d u \\
& =\left\{-u(\ln u)^{2} e^{-u}\right\}_{-q_{2}}^{-q_{1}}+2\left\{-(\ln u) e^{-u}\right\}_{-q_{2}}^{-q_{1}}+2 \int_{-q_{2}}^{-q_{1}} \frac{e^{-u}}{u} d u \\
& +\left\{-(\ln u)^{2} e^{-u}\right\}_{-q_{2}}^{-q_{1}}+2 \int_{-q_{2}}^{-q_{1}} \frac{e^{-u} \ln u}{u} d u . \\
& \int_{\lambda_{1}}^{\lambda_{2}} y^{2} e^{-y} f(y) d y=\int_{\lambda_{1}}^{\lambda_{2}} y^{2} e^{-y} e^{-y-e^{-y}} d y \\
& =\int_{\lambda_{1}}^{\lambda_{2}} y^{2} e^{-2 y} e^{-e^{-y}} d y \\
& =\int_{-q_{2}}^{-q_{1}}(\ln u)^{2} u e^{-u} d u \\
& =\left\{-u(\ln u)^{2} e^{-u}\right\}_{-q_{2}}^{-q_{1}}+2\left\{-(\ln u) e^{-u}\right\}_{-q_{2}}^{-q_{1}}+2 \int_{-q_{2}}^{-q_{1}} \frac{e^{-u}}{u} d u \\
& +\left\{-(\ln u)^{2} e^{-u}\right\}_{-q_{2}}^{-q_{1}}+2 \int_{-q_{2}}^{-q_{1}} \frac{e^{-u} \ln u}{u} d u \quad \text { [using equation( }
\end{aligned}
$$




$$
\begin{aligned}
= & q_{1}\left[\ln \left(-q_{1}\right)\right]^{2} e^{q_{1}}-q_{2}\left[\ln \left(-q_{2}\right)\right]^{2} e^{q_{2}}-2 \ln \left(-q_{1}\right) e^{q_{1}}+2 \ln \left(-q_{2}\right) e^{q_{2}}+ \\
& 2 E_{1}\left(-q_{2}\right)-2 E_{1}\left(-q_{1}\right)-\left(\ln \left(-q_{1}\right)\right)^{2} e^{q_{1}}+\left(\ln \left(-q_{2}\right)\right)^{2} e^{q_{2}} \\
& +2 \int_{-q_{2}}^{-q_{1}} \frac{e^{-u}(\ln u)}{u} d u \\
= & q_{1} r_{1}^{2} p_{1}-q_{2} r_{2}^{2}\left(1-p_{2}\right)-2 r_{1} p_{1}+2 r_{2}\left(1-p_{2}\right)+2 E_{1}\left(-q_{1}\right)-2 E_{1}\left(-q_{2}\right) \\
& -r_{1}^{2} p_{1}+r_{2}^{2}\left(1-p_{2}\right)+2 \int_{-q_{2}}^{-q_{1}} \frac{e^{-u} \ln u}{u} d u \\
= & p_{1} q_{1} r_{1}^{2}-\left(1-p_{2}\right) q_{2} r_{2}^{2}-p_{1} r_{1}^{2}+\left(1-p_{2}\right) r_{2}^{2}-2\left[p_{1} r_{1}-\left(1-p_{2}\right) r_{2}\right. \\
& \left.+E_{1}\left(-q_{1}\right)-E_{1}\left(-q_{2}\right)\right]+2 \int_{-q_{2}}^{-q_{1}} \frac{e^{-u} \ln u}{u} d u \\
& p_{1}\left(q_{1}-1\right) r_{1}^{2}+\left(1-p_{2}\right)\left(1-q_{2}\right) r_{2}^{2}-2\left\{p_{1} r_{1}-\left(1-p_{2}\right) r_{2}\right. \\
& \left.+E_{1}\left(-q_{1}\right)-E_{1}\left(-q_{2}\right)\right\}+2 \int_{-q_{2}}^{-q_{1}} \frac{\ln u}{u} e^{-u} d u .
\end{aligned}
$$

$$
\begin{aligned}
& \int_{\lambda_{1}}^{\lambda_{2}} y^{2} e^{-y} e^{-e^{-y} d y=} \int_{-q_{2}}^{-q_{1}}(\ln u)^{2} e^{-u} d u \\
&=\left\{-(\ln u)^{2} e^{-u}\right\}_{-q_{2}}^{-q_{1}}+2 \int_{-q_{2}}^{-q_{1}} \frac{(\ln u) e^{-u}}{u} d u \\
& {\left[\text { using intergration by parts formula where } U=(\ln u)^{2}, d V=e^{-u} d u\right] } \\
&=-\left(\ln \left(-q_{1}\right)\right)^{2} e^{q_{1}}+\left(\ln \left(-q_{2}\right)\right)^{2} e^{q_{2}}+2 \int_{-q_{2}}^{-q_{1}} \frac{(\ln u) e^{-u}}{u} d u \\
&=-p_{1} r_{1}^{2}+\left(1-p_{2}\right) r_{2}^{2}+2 \int_{-q_{2}}^{-q_{1}} \frac{(\ln u) e^{-u}}{u} d u .
\end{aligned}
$$


Now,

$$
2 \int_{-q_{2}}^{-q_{1}} \frac{\ln u}{u} e^{-u} d u=-2 \int_{-q_{1}}^{-q_{2}} \frac{\ln u}{u}\left\{\sum_{n=0}^{\infty} \frac{(-1)^{n} u^{n}}{n !}\right\} d u
$$

Using series expansion for $E_{1}(u)$ given by

$$
E_{1}(u)=\gamma-\ln u-\sum_{n=1}^{\infty} \frac{(-1)^{n} u^{n}}{n n !}
$$

(see Abramowitz and Stegun, 1970, page 229) and the calculations

$$
\begin{aligned}
\int_{-q_{1}}^{-q_{2}} \frac{\ln u}{u} d u & =\frac{1}{2}\left(r_{2}^{2}-r_{1}^{2}\right) \\
\text { and } \quad \int_{-q_{1}}^{-q_{2}}(\ln u) u^{n-1} d u & =\left\{\frac{u^{n} \ln u}{n}-\frac{u^{n}}{n^{2}}\right\}_{u=-q_{1}}^{u=-q_{2}}, \quad n=1,2, \ldots \infty
\end{aligned}
$$

we get

$$
\begin{aligned}
2 \int_{-q_{2}}^{-q_{1}} \frac{\ln u}{u} e^{-u} d u= & r_{2}^{2}-r_{1}^{2}+2\left\{\ln u\left(\gamma+\ln u+E_{1}(u)\right)\right\}_{u=-q_{1}}^{u=-q_{2}}+2\left\{\sum_{n=1}^{\infty} \frac{(-1)^{n} u^{n}}{n^{2} n !}\right\}_{u=-q_{1}}^{u=-q_{2}} \\
= & r_{2}^{2}-r_{1}^{2}+2\left\{r_{2}\left(\gamma+E_{1}\left(-q_{2}\right)\right)-r_{1}\left(\gamma+E_{1}\left(-q_{1}\right)\right)\right\} \\
& +2\left\{S\left(1-p_{2}\right)-S\left(p_{1}\right)\right\}
\end{aligned}
$$

where

$$
S(p)=\sum_{n=1}^{\infty} \frac{(\ln p)^{n}}{n^{2} n !}, \quad 0<p<1
$$


Finally,

$$
\begin{aligned}
\frac{\lambda_{1}^{2} f^{2}\left(\lambda_{1}\right)}{p_{1}}+\frac{\lambda_{2}^{2} f^{2}\left(\lambda_{2}\right)}{p_{2}} & =\frac{\lambda_{1}^{2} q_{1}^{2} e^{2 q_{1}}}{p_{1}}+\frac{\lambda_{2}^{2} q_{2}^{2} e^{2 q_{2}}}{p_{2}} \\
& =\frac{p_{1}^{2} q_{1}^{2} r_{1}^{2}}{p_{1}}+\frac{\left(1-p_{2}\right)^{2} q_{2}^{2} r_{2}^{2}}{p_{2}} \\
& =p_{1} q_{1}^{2} r_{1}^{2}+\frac{\left(1-p_{2}\right)^{2} q_{2}^{2} r_{2}^{2}}{p_{2}} .
\end{aligned}
$$

Using the calculations in (3.7), (3.13), (3.14), (3.17), (3.20) we get

$$
\begin{aligned}
\mathcal{I}_{22}^{*}= & \int_{\lambda_{1}}^{\lambda_{2}} L_{2}^{2}(y) f(y) d y+\frac{\lambda_{1}^{2} f^{2}\left(\lambda_{1}\right)}{p_{1}}+\frac{\lambda_{2}^{2} f^{2}\left(\lambda_{2}\right)}{p_{2}} \\
= & 1-p_{1}-p_{2}-2 p_{1} r_{1}+2\left(1-p_{2}\right) r_{2}-2 E_{1}\left(-q_{1}\right)+2 E_{1}\left(-q_{2}\right)+2 p_{1}+2 p_{2}-2 \\
& \left.+2 p_{1}\left(1-q_{1}\right) r_{1}-2\left(1-p_{2}\right)\left(1-q_{2}\right) r_{2}\right)+2 E_{1}\left(-q_{1}\right)-2 E_{1}\left(-q_{2}\right)+p_{1}\left(q_{1}-1\right) r_{1}^{2} \\
& +\left(1-p_{2}\right)\left(1-q_{2}\right) r_{2}^{2}-2\left[p_{1} r_{1}-\left(1-p_{2}\right) r_{2}+E_{1}\left(-q_{1}\right)-E_{1}\left(-q_{2}\right)\right]+2 \int_{-q_{2}}^{-q_{1}} \frac{\ln u e^{-u}}{u} d u \\
& +2\left(1-p_{1}-p_{2}\right)-p_{1} q_{1} r_{1}\left[\left(1+q_{1}\right) r_{1}-2\right]+\left(1-p_{2}\right) q_{2} r_{2}\left[\left(1+q_{2}\right) r_{2}-2\right]+p_{1} q_{1} r_{1}^{2} \\
& +\frac{\left(1-p_{2}\right)^{2} q_{2}^{2} r_{2}^{2}}{p_{2}}
\end{aligned}
$$




$$
\begin{aligned}
& =1-p_{1}-p_{2}-2 p_{1} r_{1}+2 p_{1}+2 p_{2}-2+2 p_{1} r_{1}-2 p_{1} q_{1} r_{1}-2 p_{1} r_{1}+p_{1} q_{1} r_{1}^{2}-p_{1} r_{1}^{2}+2 \\
& -2 p_{1}-2 p_{2}-p_{1} q_{1} r_{1}^{2}-p_{1} q_{1}^{2} r_{1}^{2}+2 p_{1} q_{1} r_{1} \\
& +\left(1-p_{2}\right)\left[-2 r_{2}-2 r_{2}+2 q_{2} r_{2}+r_{2}^{2}-q_{2} r_{2}^{2}+2 r_{2}+q_{2} r_{2}^{2}+q_{2}^{2} r_{2}^{2}-2 q_{2} r_{2}+\frac{\left(1-p_{2}\right) q_{2}^{2} r_{2}^{2}}{p_{2}}\right] \\
& +p_{1} q_{1}^{2} r_{1}^{2}+2 E_{1}\left(-q_{1}\right)-2 E_{1}\left(-q_{2}\right)+2 E_{1}\left(-q_{1}\right)-2 E_{1}\left(-q_{2}\right)-2 E_{1}\left(-q_{1}\right)+2 E_{1}\left(-q_{2}\right) \\
& +r_{2}^{2}-r_{1}^{2}+2\left[r_{2}\left(\gamma+E_{1}\left(-q_{2}\right)-r_{1}\left(\gamma+E_{1}\left(-q_{1}\right)\right)\right]+2\left[S\left(1-p_{2}\right)-S\left(p_{1}\right)\right]\right. \\
& =1-p_{1}-p_{2}-p_{1} r_{1}\left(2+r_{1}\right)+\left(1-p_{2}\right)\left(r_{2}^{2}+2 r_{2}+\frac{q_{2}^{2} r_{2}^{2}}{p_{2}}\right)+2\left[\left(1+r_{2}\right) E_{1}\left(-q_{2}\right)-\left(1+r_{1}\right)\right. \\
& \left.E_{1}\left(-q_{1}\right)\right]+\left(r_{2}-r_{1}\right)\left(r_{2}+r_{1}\right)+2 r_{2} \gamma-2 r_{1} \gamma+2\left[S\left(1-p_{2}\right)-S\left(p_{1}\right)\right] \\
& =1-p_{1}-p_{2}-p_{1} r_{1}\left(2+r_{1}\right)+\left(1-p_{2}\right) r_{2}\left(2+r_{2}+q_{2}^{2} r_{2} / p_{2}\right)+\left(r_{2}-r_{1}\right)\left(2 \gamma+r_{1}+r_{2}\right) \\
& +2\left[\left(1+r_{2}\right) E_{1}\left(-q_{2}\right)-\left(1+r_{1}\right) E_{1}\left(-q_{1}\right)\right]+2\left[S\left(1-p_{2}\right)-S\left(p_{1}\right)\right], \\
& \text { where } E_{1}(u)=\int_{u}^{\infty} \frac{e^{-t}}{t} d t \quad(u>0) \text {, and } S(p)=\sum_{n=1}^{\infty} \frac{[\ln p]^{n}}{n^{2} n !} \text {. }
\end{aligned}
$$

Values of $E_{1}(u)$ for different values of $u$ can be obtained from the package Maple. Also, values of the infinite sum $S(p)$ for different values of $p$ can be obtained from the same package. Tabulated values of $E_{1}(u)$ are also available in Abramowitz and Stegun (1970) (p. 238-243). In Table 3.1 we have given the values of the infinite sums $(-1) S(p)$ and $(-1) S(1-p)$ for $p=0.01(0.01) 0.50$. 
Table 3.1: Values of the infinite sums $(-1) S(p)$ and $(-1) S(1-p)$ for different values of $p$.

\begin{tabular}{ccc|ccc}
\hline$p$ & $(-1) S(p)$ & $(-1) S(1-p)$ & $p$ & $(-1) S(p)$ & $(-1) S(1-p)$ \\
\hline 0.01 & 3.036413535 & 0.010037728 & 0.26 & 1.158216434 & 0.290256995 \\
0.02 & 2.705968837 & 0.020151841 & 0.27 & 1.130065261 & 0.302883055 \\
0.03 & 2.498943550 & 0.030343758 & 0.28 & 1.102741323 & 0.315642094 \\
0.04 & 2.345082708 & 0.040614943 & 0.29 & 1.076190787 & 0.328537500 \\
0.05 & 2.221384502 & 0.050966900 & 0.30 & 1.050365058 & 0.341572798 \\
0.06 & 2.117289324 & 0.061401182 & 0.31 & 1.025220108 & 0.354751659 \\
0.07 & 2.027029221 & 0.071919386 & 0.32 & 1.000715918 & 0.368077903 \\
0.08 & 1.947092564 & 0.082523158 & 0.33 & 0.976816000 & 0.381555512 \\
0.09 & 1.875175366 & 0.093214197 & 0.34 & 0.953486982 & 0.395188638 \\
0.10 & 1.809680652 & 0.103994253 & 0.35 & 0.930698269 & 0.408981613 \\
0.11 & 1.749453891 & 0.114865133 & 0.36 & 0.908421731 & 0.422938958 \\
0.12 & 1.693632101 & 0.125828701 & 0.37 & 0.886631442 & 0.437065399 \\
0.13 & 1.641552509 & 0.136886880 & 0.38 & 0.865303461 & 0.451365875 \\
0.14 & 1.592694533 & 0.148041659 & 0.39 & 0.844415620 & 0.465845554 \\
0.15 & 1.546641471 & 0.159295091 & 0.40 & 0.823947360 & 0.480509844 \\
0.16 & 1.503054328 & 0.170649297 & 0.41 & 0.803879574 & 0.495364415 \\
0.17 & 1.461653449 & 0.182106472 & 0.42 & 0.784194470 & 0.510415210 \\
0.18 & 1.422205298 & 0.193668885 & 0.43 & 0.764875453 & 0.525668466 \\
0.19 & 1.384512745 & 0.205338885 & 0.44 & 0.745907019 & 0.541130735 \\
0.20 & 1.348407794 & 0.217118903 & 0.45 & 0.727274659 & 0.556808901 \\
0.21 & 1.313746058 & 0.229011456 & 0.46 & 0.708964774 & 0.572710212 \\
0.22 & 1.280402481 & 0.241019153 & 0.47 & 0.690964603 & 0.588842297 \\
0.23 & 1.248268001 & 0.253144698 & 0.48 & 0.673262150 & 0.605213200 \\
0.24 & 1.217246902 & 0.265390895 & 0.49 & 0.655846126 & 0.621831410 \\
0.25 & 1.187254701 & 0.277760654 & 0.50 & 0.638705891 & 0.638705891 \\
\hline & & & & &
\end{tabular}

The above expressions for $\mathcal{I}_{i j}^{*}$ are much simpler than the symbolic formulas given in Harter and Moore (1968). We have verified that the above expressions for $\mathcal{I}_{i j}^{*}$, $i, j=1,2$ accurately give the elements of the asymptotic covariance matrix of the maximum likelihood estimators for all values of $p_{1}$ and $p_{2}$ tabulated by Harter and Moore (1968). The expression for $\mathcal{I}_{11}^{*}$ given in Harter and Moore (1968) (see relation 
(3.5), p.893) appears to be incorrect.

We now construct an estimator $\left(T_{1 n}^{*}, T_{2 n}^{*}\right)$ of $(\theta, \sigma)$ defined by

$$
T_{i n}^{*}=n^{-1} \sum_{j=s}^{l} J_{i}^{*}\left(\frac{j}{n+1}\right) X_{j n}+a_{i} X_{s n}+b_{i} X_{l n}
$$

where $X_{s n}$ and $X_{l n}$ are the smallest and largest available order statistics, and

$$
\left\{J_{1}^{*}(u), J_{2}^{*}(u)\right\}=\left\{L_{1}^{\prime}(y), L_{2}^{\prime}(y)\right\} \mathcal{I}^{*-1}, \quad \lambda_{1}<y=F^{-1}(u)<\lambda_{2}
$$

where $\mathcal{I}^{*-1}=\left(\left(\mathcal{I}^{* i j}\right)\right)_{2 \times 2}$ is the inverse of the matrix $\mathcal{I}^{*}$. After simplifying we have

$$
\begin{aligned}
\left\{J_{1}^{*}(u), J_{2}^{*}(u)\right\} & =\left[e^{\ln (-\ln u)}, 1-e^{\ln (-\ln u)}-\ln (-\ln u) e^{\ln (-\ln u}\right] \mathcal{I}^{*-1} \\
& =[-\ln u, 1+\ln u+\ln (-\ln u) \ln u]\left(\begin{array}{ll}
\mathcal{I}^{* 11} & \mathcal{I}^{* 12} \\
\mathcal{I}^{* 21} & \mathcal{I}^{* 22}
\end{array}\right) \\
J_{1}^{*}(u) & =-(\ln u) \mathcal{I}^{* 11}+(1+\ln u+\ln (-\ln u) \ln u) \mathcal{I}^{* 21} \\
& =\mathcal{I}^{* 12}+\ln u\left[\{1+\ln (-\ln u)\} \mathcal{I}^{* 12}-\mathcal{I}^{* 11}\right] \\
J_{2}^{*}(u) & =-(\ln u) \mathcal{I}^{* 12}+(1+\ln u+\ln (-\ln u) \ln u) \mathcal{I}^{* 22} \\
& =\mathcal{I}^{* 22}+\ln u\left[\{1+\ln (-\ln u)\} \mathcal{I}^{* 22}-\mathcal{I}^{* 12}\right]
\end{aligned}
$$


and

$$
\begin{aligned}
& a_{i}=f\left(\lambda_{1}\right)\left[L_{1}\left(\lambda_{1}\right)+\frac{f\left(\lambda_{1}\right)}{p_{1}}\right] \mathcal{I}^{* i 1}+f\left(\lambda_{1}\right)\left[L_{2}\left(\lambda_{1}\right)+\frac{\lambda_{1} f\left(\lambda_{1}\right)}{p_{1}}\right] \mathcal{I}^{* i 2}, \quad i=1,2 \\
& =e^{-\lambda_{1}-e^{-\lambda_{1}}}\left[1-e^{-\lambda_{1}}+\frac{e^{-\lambda_{1}-e^{-\lambda_{1}}}}{p_{1}}\right] \mathcal{I}^{* i 1}+ \\
& e^{-\lambda_{1}-e^{-\lambda_{1}}}\left[-1+\lambda_{1}-\lambda_{1} e^{-\lambda_{1}}+\frac{\lambda_{1} e^{-\lambda_{1}-e^{-\lambda_{1}}}}{p_{1}}\right] \mathcal{I}^{* i 2} \\
& =e^{r_{1}-e^{r_{1}}}\left[1-e^{r_{1}}+\frac{e^{r_{1}-e^{r_{1}}}}{p_{1}}\right] \mathcal{I}^{* i 1}+e^{r_{1}-e^{r_{1}}}\left[-1-r_{1}+r_{1} e^{r_{1}}+\frac{-r_{1} e^{r_{1}-e^{r_{1}}}}{p_{1}}\right] \mathcal{I}^{* i 2}, \\
& {\left[\lambda_{1}=-r_{1}\right]} \\
& =e^{\ln \left(-q_{1}\right)-e^{\ln \left(-q_{1}\right)}}\left[1-e^{\ln \left(-q_{1}\right)}+\frac{e^{\ln \left(-q_{1}\right)-e^{\ln \left(-q_{1}\right)}}}{p_{1}}\right] \mathcal{I}^{* i 1}+ \\
& e^{\ln \left(-q_{1}\right)-e^{\ln \left(-q_{1}\right)}}\left[-1-r_{1}+r_{1} e^{\ln \left(-q_{1}\right)}+\frac{-r_{1} e^{\ln \left(-q_{1}\right)-e^{\ln \left(-q_{1}\right)}}}{p_{1}}\right] \mathcal{I}^{* i 2}, \\
& {\left[r_{1}=\ln \left(-q_{1}\right)\right]} \\
& =-q_{1} e^{q_{1}}\left[1+q_{1}-\frac{q_{1} e^{q_{1}}}{p_{1}}\right] \mathcal{I}^{* i 1}-q_{1} e^{q_{1}}\left[-1-r_{1}-r_{1} q_{1}+\frac{r_{1} q_{1} e^{q_{1}}}{p_{1}}\right] \mathcal{I}^{* i 2} \\
& =-q_{1} p_{1}\left[1+q_{1}-q_{1}\right] \mathcal{I}^{* i 1}-q_{1} p_{1}\left[-1-r_{1}-r_{1} q_{1}+r_{1} q_{1}\right] \mathcal{I}^{* i 2} \\
& {\left[q_{1}=\ln \left(p_{1}\right)\right]} \\
& =p_{1} q_{1}\left[\left(1+r_{1}\right) \mathcal{I}^{* i 2}-\mathcal{I}^{* i 1}\right]
\end{aligned}
$$




$$
\begin{aligned}
& b_{i}=f\left(\lambda_{2}\right)\left[-L_{1}\left(\lambda_{2}\right)+\frac{f\left(\lambda_{2}\right)}{p_{2}}\right] \mathcal{I}^{* i 1}+f\left(\lambda_{2}\right)\left[-L_{2}\left(\lambda_{2}\right)+\frac{\lambda_{2} f\left(\lambda_{2}\right)}{p_{2}}\right] \mathcal{I}^{* i 2}, \quad i=1,2 \\
& =e^{-\lambda_{2}-e^{-\lambda_{2}}}\left[-1+e^{-\lambda_{2}}+\frac{e^{-\lambda_{2}-e^{-\lambda_{2}}}}{p_{2}}\right] \mathcal{I}^{* i 1}+ \\
& e^{-\lambda_{2}-e^{-\lambda_{2}}}\left[1-\lambda_{2}+\lambda_{2} e^{-\lambda_{2}}+\frac{\lambda_{2} e^{-\lambda_{2}-e^{-\lambda_{2}}}}{p_{2}}\right] \mathcal{I}^{* i 2} \\
& =e^{r_{2}-e^{r_{2}}}\left[-1+e^{r_{2}}+\frac{e^{r_{2}-e^{r_{2}}}}{p_{2}}\right] \mathcal{I}^{* i 1}+e^{r_{2}-e^{r_{2}}}\left[1+r_{2}-r_{2} e^{r_{2}}-\frac{r_{2} e^{r_{2}-e^{r_{2}}}}{p_{2}}\right] \mathcal{I}^{* i 2}, \\
& {\left[\lambda_{2}=-r_{2}\right]} \\
& =e^{\ln \left(-q_{2}\right)-e^{\ln \left(-q_{2}\right)}}\left[-1+e^{\ln \left(-q_{2}\right)}+\frac{e^{\ln \left(-q_{2}\right)-e^{\ln \left(-q_{2}\right)}}}{p_{2}}\right] \mathcal{I}^{* i 1}+ \\
& e^{\ln \left(-q_{2}\right)-e^{\ln \left(-q_{2}\right)}}\left[1+r_{2}-r_{2} e^{\ln \left(-q_{2}\right)}-\frac{r_{2} e^{\ln \left(-q_{2}\right)-e^{\ln \left(-q_{2}\right)}}}{p_{2}}\right] \mathcal{I}^{* i 2}, \\
& {\left[r_{2}=\ln \left(-q_{2}\right)\right]} \\
& =-q_{2} e^{q_{2}}\left[-1-q_{2}-\frac{q_{2} e^{q_{2}}}{p_{2}}\right] \mathcal{I}^{* i 1}-q_{2} e^{q_{2}}\left[1+r_{2}+r_{2} q_{2}+\frac{r_{2} q_{2} e^{q_{2}}}{p_{2}}\right] \mathcal{I}^{* i 2} \\
& =-q_{2}\left(1-p_{2}\right)\left[-1-q_{2}-\frac{q_{2}\left(1-p_{2}\right)}{p_{2}}\right] \mathcal{I}^{* i 1}-q_{2}\left(1-p_{2}\right)\left[1+r_{2}+r_{2} q_{2}+\frac{r_{2} q_{2}\left(1-p_{2}\right)}{p_{2}}\right] \mathcal{I}^{* i 2} \\
& {\left[q_{2}=\ln \left(1-p_{2}\right)\right]} \\
& =\frac{\left(1-p_{2}\right) q_{2}}{p_{2}}\left[\left(p_{2}+q_{2}\right) \mathcal{I}^{* i 1}-\left\{p_{2}+r_{2}\left(p_{2}+q_{2}\right)\right\} \mathcal{I}^{* i 2}\right] .
\end{aligned}
$$


We have, $\quad J_{i}^{*}(u)=\mathcal{I}^{* i 2}+\ln u\left[\{1+\ln (-\ln u)\} \mathcal{I}^{* i 2}-\mathcal{I}^{* i 1}\right], \quad i=1,2$

$$
\begin{aligned}
& a_{i}=p_{1} q_{1}\left[\left(1+r_{1}\right) \mathcal{I}^{* i 2}-\mathcal{I}^{* i 1}\right], \quad i=1,2 \\
& b_{i}=\frac{\left(1-p_{2}\right) q_{2}}{p_{2}}\left[\left(p_{2}+q_{2}\right) \mathcal{I}^{* i 1}-\left\{p_{2}+r_{2}\left(p_{2}+q_{2}\right)\right\} \mathcal{I}^{* i 2}\right], \quad i=1,2 .
\end{aligned}
$$

The estimator $\left(T_{1 n}^{*}, T_{2 n}^{*}\right)$ is asymptotically normally distributed with mean vector $(\theta, \sigma)$ and covariance matrix $\sigma^{2}\left(n \mathcal{I}^{*}\right)^{-1}$. The estimators are asymptotically efficient and can be justified similarly as given in the previous chapter.

We present the necessary calculations for the estimators $T_{i n}^{*}, i=1,2$ in two special cases.

Case 1. With $p_{1}=p_{2}=0.05$ we have

$$
\begin{gathered}
\mathcal{I}^{*}=\left(\begin{array}{cc}
0.9499890 & -0.3054810 \\
-0.3054810 & 1.4883838
\end{array}\right) \\
J_{1}^{*}(u)=0.2313+\ln u[-0.8957+0.2313 \ln (-\ln u)], \quad 0.05<u<0.95 \\
J_{2}^{*}(u)=0.7193+\ln u[0.4880+0.7193 \ln (-\ln u)], \quad 0.05<u<0.95 \\
a_{1}=0.0962, \quad b_{1}=0.0136, \quad a_{2}=-0.1913, \quad b_{2}=0.0380 .
\end{gathered}
$$

Case 2. With $p_{1}=p_{2}=0.1$ we have

$$
\mathcal{I}^{*}=\left(\begin{array}{cc}
0.8999075 & -0.2098484 \\
-0.2098484 & 1.2362301
\end{array}\right)
$$




$$
\begin{aligned}
J_{1}^{*}(u) & =0.1964+\ln u[-0.9606+0.1964 \ln (-\ln u)], \quad 0.1<u<0.9 \\
J_{2}^{*}(u) & =0.8423+\ln u[0.6458+0.8423 \ln (-\ln u)], \quad 0.1<u<0.9 \\
a_{1} & =0.1835, \quad b_{1}=0.0268, \quad a_{2}=-0.3105, \quad b_{2}=0.0905 .
\end{aligned}
$$

Example 2. We consider the sample in Example 1 and assume type II censoring where the observations which lie in the sample percentile ranges $(0,0.1)$ and $(0.9,1)$ are censored.

Based on the middle 40 observations the estimates of $\theta$ and $\sigma$ are $T_{1 n}^{*}=4.9132$ and $T_{2 n}^{*}=1.7695$ respectively.

Using the formulas for the elements of the information matrix developed in this thesis, we have given in Table 3.2, the element of $\mathcal{I}^{*}$ matrix for different degree of censoring. We also provide the elements of $\mathcal{I}^{*-1}$ in table 3.3. Obviously, the elements of the inverse of the information matrix multiplied by $\sigma^{2} / n$ are the variances and covariances of our estimators. If the left censoring proportion is replaced by $p_{2}$ and the right censoring proportion is replaced by $p_{1}$ (as we consider the distribution of largest values and Harter and Moore (1968) consider the distribution of smallest values), then our Table 3.3 completely agrees with Table 1 of Harter and Moore (1968). Also note that sign of the covariances between our estimators is opposite to that of Harter and Moore (1968) as we are considering the distribution of largest values (lines 20-23 of Page 893 of Harter and Moore (1968)). 
Table 3.2: Elements of the matrix $\mathcal{I}^{*}=\left(\left(\mathcal{I}_{i j}^{*}\right)\right)_{2 \times 2}$

\begin{tabular}{|c|c|c|c|c|}
\hline & & $\mathcal{I}^{* 11}$ & $\mathcal{I}^{*}$ & $\mathcal{I}^{* 22}$ \\
\hline 01 & 0.0 & 1.000000 & & 823681 \\
\hline 0.0 & 0.1 & 999908 & -0.425641 & 708600 \\
\hline & 0 . & 9172 & 35313 & .57629 \\
\hline & 0 & & -0.453275 & \\
\hline & 0 . & 901414 & -0.480891 & \\
\hline & & & -0.519322 & \\
\hline & & & & \\
\hline & & & -0.628421 & .942522 \\
\hline & & & & \\
\hline & 0. & 0.689100 & 07121 & 088215 \\
\hline & & & & \\
\hline & & & $884 \overline{8}$ & 236230 \\
\hline & & & -0.219520 & 925 \\
\hline & 0 & & -0.237481 & 0.963122 \\
\hline & & & -0.265099 & 0.82078 \\
\hline & & & 3304 & 62317 \\
\hline & & & & $044^{2}$ \\
\hline & & & 28 & 0.47 \\
\hline & & & & \\
\hline & & & & 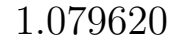 \\
\hline & & & & 0.96 \\
\hline & & & & \\
\hline & & & & \\
\hline & & & & \\
\hline & U. & & & \\
\hline & & & & \\
\hline & & & -0 & 0.1 \\
\hline & 0 & & & \\
\hline & & & & 0.7 \\
\hline & & & & \\
\hline & & & & \\
\hline & & & & \\
\hline & & & & \\
\hline & & & & \\
\hline & & & & \\
\hline & & & & \\
\hline & & & & \\
\hline & & & & \\
\hline & & & & \\
\hline & & & & \\
\hline & & & & \\
\hline & & & & \\
\hline & & & & \\
\hline & & & & \\
\hline & & & & \\
\hline & & & & \\
\hline & & & & \\
\hline & & & & \\
\hline & & & & \\
\hline & & & & \\
\hline & & & & \\
\hline & & & & \\
\hline & 0 & & & \\
\hline & U. & & & \\
\hline & & & & \\
\hline
\end{tabular}


Table 3.3: Elements of the matrix $\mathcal{I}^{*-1}=\left(\left(\mathcal{I}^{* i j}\right)\right)_{2 \times 2}$.

\begin{tabular}{|c|c|c|c|c|}
\hline & & $\mathcal{I}^{* 11}$ & $\mathcal{I}^{* 12}$ & $\mathcal{I}^{* 22}$ \\
\hline 0.0 & 0.0 & 1.108665 & 0.257022 & 0.607927 \\
\hline 0.0 & 0.1 & .118727 & 0.278694 & 0.654702 \\
\hline 0.0 & 0.2 & .137715 & 0.314194 & 0.721168 \\
\hline 0.0 & 0.3 & 1.171354 & 0.369870 & 0.813416 \\
\hline 0.0 & 0.4 & .230645 & 0.457647 & 0.943493 \\
\hline 0.0 & 0.5 & 1.338098 & 0.600672 & 1.134038 \\
\hline 0.0 & 0.6 & 1.545203 & 0.848735 & 1.431430 \\
\hline 0.0 & 0.7 & 1.991079 & 1.327539 & 1.946111 \\
\hline 0.0 & 0.8 & 3.170376 & 2.450529 & 3.016820 \\
\hline 0.0 & 0.9 & 8.177861 & 6.555257 & 6.388191 \\
\hline 0.1 & 0.0 & 1.151684 & 0.176413 & 0.767044 \\
\hline 0.1 & 0.1 & 1.157024 & 0.196403 & 0.842250 \\
\hline 0.1 & 0.2 & 1.168880 & 0.232437 & 0.952080 \\
\hline 0.1 & $0 . \overline{3}$ & 1.192915 & 0.294142 & 1.110818 \\
\hline 0.1 & 0.4 & 1.241015 & 0.400827 & 1.347812 \\
\hline 0.1 & 0.5 & 1.340734 & 0.594518 & 1.724528 \\
\hline 0.1 & 0.6 & 1.566598 & 0.981853 & 2.389557 \\
\hline 0.1 & 0.7 & 2.178199 & 1.911687 & 3.804751 \\
\hline 0.1 & 0.8 & 4.683862 & 5.292215 & 8.369956 \\
\hline 0.2 & 0.0 & 1.252617 & 0.049288 & 0.928191 \\
\hline 0.2 & 0.1 & 1.253484 & 0.058920 & 1.039534 \\
\hline 0.2 & 0.2 & 1.257014 & 0.083088 & 1.207078 \\
\hline 0.2 & 0.3 & .267204 & 0.133736 & 1.460390 \\
\hline 0.2 & 0.4 & 1.293682 & 0.236991 & 1.864610 \\
\hline 0.2 & 0.5 & 1.363071 & 0.459023 & 2.576961 \\
\hline $0 . \overline{2}$ & 0.6 & 1.568133 & 1.013806 & 4.080712 \\
\hline $0 . \overline{2}$ & 0.7 & 2.432859 & 3.041565 & 8.841320 \\
\hline $0 . \overline{3}$ & 0.0 & 1.447258 & -0.144825 & 1.122447 \\
\hline 0.3 & 0.1 & 1.448869 & -0.160918 & 1.287741 \\
\hline 0.3 & 0.2 & 1.449409 & -0.172088 & 1.546735 \\
\hline 0.3 & 0.3 & 1.449526 & -0.168598 & 1.963716 \\
\hline 0.3 & 0.4 & 1. 452168 & -0.125731 & 2.698811 \\
\hline 0.3 & 0.5 & 1.469964 & 0.038778 & 4.242607 \\
\hline 0.3 & 0.6 & .581070 & 0.769896 & 9.078796 \\
\hline 0.4 & 0.0 & 1.811959 & -0.446603 & 1.372782 \\
\hline 0.4 & 0.1 & 1.834013 & -0.521153 & 1.625480 \\
\hline 0.4 & 0.2 & 1.859086 & -0.623606 & 2.045511 \\
\hline 0.4 & 0.3 & .888035 & -0.770185 & 2.790888 \\
\hline 0.4 & 0.4 & .924836 & -1.009384 & 4.354570 \\
\hline 0.4 & 0.5 & 1.989349 & -1.568276 & 9.226890 \\
\hline 0.5 & 0.0 & 2.510236 & -0.935766 & 1.716182 \\
\hline 0.5 & 0.1 & 2.619087 & -1.146524 & 2.124585 \\
\hline 0.5 & 0.2 & 2.782556 & -1.495475 & 2.870038 \\
\hline 0.5 & 0.5 & 3.067 & .164654 & 4.44 \\
\hline 0.5 & 0.4 & 3.813216 & -4.073545 & 9.331396 \\
\hline 0.6 & 0.0 & 3.933022 & -1.785526 & 2.224740 \\
\hline 0.6 & 0. & 4.368319 & -2.347534 & 2.950577 \\
\hline 0.6 & 0.2 & 5.203882 & $-\overline{3} .491862$ & 4.518143 \\
\hline 0.6 & 0. & 7.56 & -6.891443 & 9.412787 \\
\hline 0.7 & 0.0 & 7.190428 & -3.438603 & 3.065517 \\
\hline 0.7 & 0.1 & 9.027691 & -5.117493 & 4.599897 \\
\hline 0.7 & 0.2 & 14.464851 & -10.270577 & 9.484081 \\
\hline 0.8 & 0.0 & 16.478776 & -7.375313 & 4.738767 \\
\hline 0.8 & 0.1 & 27.763081 & -14.752839 & 9.562343 \\
\hline 0.9 & 0.0 & 60.517148 & -22.187224 & 9.744670 \\
\hline
\end{tabular}




\section{A simulation study}

We have also performed a Monte Carlo simulation study to find the means, variances and covariances of the estimators from 100000 samples for sample sizes $n=10, n=16, n=20, n=24, n=28$ and $n=30$ for different degrees of censoring. The results are given in Tables 3.4, 3.5, 3.6, 3.7, 3.8 and 3.9 for $n=10, n=16$, $n=20, n=24, n=28$ and $n=30$ respectively. The notations for all tables are described as follows. First, note that $\hat{\theta}$ and $\hat{\sigma}$ denote the estimators $T_{1 n}^{*}, T_{2 n}^{*}$ of $\theta$ and

$\sigma$ respectively. The notations $M(\hat{\theta}), M(\hat{\sigma}), V(\hat{\theta}), \operatorname{Cov}(\hat{\theta}, \hat{\sigma})$ and $V(\hat{\sigma})$ are described as follows.

$M(\hat{\theta})=$ mean of $\hat{\theta}$ in 100000 samples of sizes $n=10,16,20,24,28,30$.

$M(\hat{\sigma})=$ mean of $\hat{\sigma}$ in 100000 samples of sizes $n=10,16,20,24,28,30$.

$V(\hat{\theta})=$ variance of $\hat{\theta}$ in 100000 samples of sizes $n=10,16,20,24,28,30$.

$V(\hat{\sigma})=$ variance of $\hat{\sigma}$ in 100000 samples of sizes $n=10,16,20,24,28,30$.

$\operatorname{Cov}(\hat{\theta}, \hat{\sigma})=$ covariance of $\hat{\theta}$ and $\hat{\sigma}$ in 100000 samples of sizes $n=10,16,20,24,28,30$.

We used the software $\mathrm{R}$ to perform this simulation.

From all tables we note that the bias of the estimators increases as the degree of censoring increases, and the bias decreases as the sample size increases. As expected, the variances of the estimators decrease as the sample size increases. 
Table 3.4: Means, variances and covariances of the proposed estimators of parameters of type I (maximum) extreme value distribution $(\theta=0, \sigma=1)$ from 100000 samples of size $n=10$.

\begin{tabular}{|c|c|c|c|c|c|c|}
\hline$F$ & $P$ & $M(\hat{\theta})$ & $M(\hat{\sigma})$ & $V(\hat{\theta})$ & $\operatorname{Cov}(\hat{\theta}, \hat{\sigma})$ & $V(\hat{\sigma})$ \\
\hline 0.0 & 0.0 & 0.0994 & 0.8133 & 0.1138 & 0.0442 & 0.0476 \\
\hline 0.0 & 0.1 & 0.0839 & 0.7768 & 0.1135 & 0.0452 & 0.0492 \\
\hline 0.0 & 0.2 & 0.0635 & 0.6415 & 0.1151 & 0.0482 & 0.0517 \\
\hline 0.0 & 0.3 & 0.0311 & 0.5980 & 0.1201 & 0.0540 & 0.0562 \\
\hline 0.0 & 0.4 & -0.0160 & 0.5388 & 0.1286 & 0.0629 & 0.0625 \\
\hline 0.0 & 0.5 & -0.0861 & 0.4542 & 0.1446 & 0.0765 & 0.0716 \\
\hline 0.0 & 0.6 & 970 & 0.3298 & 0.1720 & 0.0982 & 0.0853 \\
\hline 0.0 & 0.7 & 1005 & 0.1259 & 0.2221 & 0.1338 & .1054 \\
\hline 0.0 & 0.8 & -0.8326 & 0.2587 & 0.3442 & 0.2091 & 0.1437 \\
\hline 0.1 & 0.0 & 54 & 0.8682 & 0.1209 & 0.0266 & 0.0685 \\
\hline 0.1 & 0.1 & 0.0900 & & 0.1200 & 0.0267 & 721 \\
\hline 0.1 & 0.2 & 0.0735 & 0.8160 & 0.1206 & 0.0291 & 0.0786 \\
\hline 0.1 & 0.3 & 0.0513 & 0.7873 & 0.1225 & 0.0351 & 0.0900 \\
\hline 0.1 & 0.4 & 0.0290 & 0.7467 & 0.1275 & 0.0450 & 054 \\
\hline 0.1 & 0.5 & -0.0118 & 0.6854 & 0.1380 & 0.0605 & 0.1258 \\
\hline 0.1 & 0.6 & -0.0823 & 0.5761 & 0.1598 & 854 & 538 \\
\hline 0.1 & 0.7 & -0.2329 & 0.3711 & 0.2032 & 0.1262 & 0.1859 \\
\hline 0.2 & 0.0 & 0.1303 & 0.8785 & 0.1349 & 0.0116 & 0.0832 \\
\hline 0.2 & 0.1 & 0.1090 & 0.8458 & 0.1327 & 0.0104 & .0880 \\
\hline 0.2 & 0.2 & 0.0938 & 0.8205 & 0.1321 & 24 & 998 \\
\hline 0.2 & 0.3 & 0.0756 & 0.7866 & 0.1332 & 0.0177 & 0.1157 \\
\hline 0.2 & 0.4 & & 0.7374 & 0.1355 & 0.0264 & 398 \\
\hline 0.2 & 0.5 & $0 .($ & 0.6503 & 0.1409 & 07 & 08 \\
\hline 0.2 & 0.6 & -0.0547 & 0.4725 & 0.1581 & 0.0667 & 0.2036 \\
\hline 0.3 & 0.0 & & 0.8644 & 0.1576 & -0.0073 & 975 \\
\hline 0.3 & 0.1 & 0 . & 0.8247 & 0.1579 & -0.0103 & 061 \\
\hline 0.3 & 0.2 & 0 . & 0.7919 & 0.1553 & -0.0108 & 215 \\
\hline 0.3 & 0.3 & 0.1205 & 0.7449 & 53 & 093 & 88 \\
\hline 0.3 & 0.4 & 0.1054 & 0.6664 & 0.1549 & 29 & 01 \\
\hline 0.3 & 0.5 & 0.0757 & 0.4974 & 0.1580 & 0.0104 & 11 \\
\hline 0.4 & 0.0 & 0.2139 & 0.8375 & 0.1996 & -0.0341 & 0.1170 \\
\hline 0.4 & 0.1 & 0.2041 & 0.7885 & 0.2029 & 406 & \\
\hline 0.4 & 0.2 & 0.1953 & 0.7 & 0.1986 & 54 & 0. \\
\hline 0.4 & 0.3 & 0.1978 & 0.6626 & 0.1969 & -0.0503 & 0.1846 \\
\hline 0.4 & 0.4 & 0.2 & 0.4964 & 0.1957 & & 0.2163 \\
\hline 0.5 & 0.0 & 0.3015 & 0.7882 & 0.2743 & -0.0744 & 0.1377 \\
\hline 0.5 & 0.1 & 0.3020 & 0.7250 & 0.2809 & -0.0876 & 0.1566 \\
\hline 0.5 & 0.2 & 0.3179 & 0.6470 & 0.2811 & 29 & 007 \\
\hline 0.5 & 0.3 & & 0.4 & 0.2810 & -0.1113 & 0.2200 \\
\hline 0.6 & 0.0 & 0.4429 & 0.7240 & 0.4109 & -0.1343 & 0.1668 \\
\hline 0.6 & 0.1 & 0.4848 & 0.6226 & 0.4349 & 631 & 0.1942 \\
\hline 0.6 & 0.2 & 0.5754 & & 0.4428 & -0.1838 & 0.2248 \\
\hline 0. & 0.0 & 0.7089 & 0.6142 & 0.6860 & -0.2304 & 0.2012 \\
\hline 0. & 0.1 & 0.8642 & 0.4300 & 0.7285 & -0.2681 & 0.2258 \\
\hline 0.8 & 0.0 & 0.9196 & 0.5051 & 0.9157 & -0.3780 & 0.2292 \\
\hline
\end{tabular}


Table 3.5: Means, variances and covariances of the proposed estimators of parameters of type I (maximum) extreme value distribution $(\theta=0, \sigma=1)$ from 100000 samples of size $n=16$.

\begin{tabular}{cc|ccccc}
\hline$p_{1}$ & $p_{2}$ & $M(\hat{\theta})$ & $M(\hat{\sigma})$ & $V(\hat{\theta})$ & $\operatorname{Cov}(\hat{\theta}, \hat{\sigma})$ & $V(\hat{\sigma})$ \\
\hline 0.0 & 0.0 & 0.0830 & 0.7770 & 0.0697 & 0.0244 & 0.0308 \\
0.0 & 0.1 & 0.0290 & 0.6948 & 0.0680 & 0.0219 & 0.0277 \\
0.0 & 0.2 & -0.0251 & 0.6281 & 0.0655 & 0.0203 & 0.0262 \\
0.0 & 0.3 & -0.0022 & 0.6648 & 0.0722 & 0.0285 & 0.0352 \\
0.0 & 0.4 & -0.0728 & 0.5777 & 0.0720 & 0.0295 & 0.0354 \\
0.0 & 0.5 & -0.0744 & 0.5764 & 0.0877 & 0.0438 & 0.0476 \\
0.0 & 0.6 & -0.2031 & 0.4312 & 0.0964 & 0.0513 & 0.0514 \\
0.0 & 0.7 & -0.4253 & 0.1943 & 0.1147 & 0.0660 & 0.0583 \\
0.0 & 0.8 & -0.6966 & -0.0323 & 0.1962 & 0.1191 & 0.0909 \\
0.5 & 0.0 & 3.2755 & -1.1429 & 1.6503 & -0.7951 & 0.4338 \\
0.5 & 0.1 & 0.2593 & 0.6677 & 0.1713 & -0.0571 & 0.0876 \\
0.5 & 0.2 & 0.3164 & 0.4641 & 0.1760 & -0.0689 & 0.0916 \\
0.5 & 0.3 & 0.2671 & 0.5748 & 0.1807 & -0.0886 & 0.1658 \\
\hline
\end{tabular}


Table 3.6: Means, variances and covariances of the proposed estimators of parameters of type I (maximum) extreme value distribution $(\theta=0, \sigma=1)$ from 100000 samples of size $n=20$.

\begin{tabular}{|c|c|c|c|c|c|c|}
\hline & & $M(\hat{\theta})$ & $M(\hat{\sigma})$ & $V(\hat{\theta})$ & $\operatorname{Cov}(\hat{\theta}, \hat{\sigma})$ & $V(\hat{\sigma})$ \\
\hline 0.0 & 0.0 & 0.0597 & 0.9045 & 0.0555 & 0.0186 & 0.0251 \\
\hline 0.0 & 0.1 & 0.0517 & 0.8807 & 0.0562 & 0.0193 & 0.0262 \\
\hline 0.0 & 0.2 & 0.0326 & 0.7573 & 0.0572 & 0.0212 & 0.0281 \\
\hline 0.0 & 0.3 & 0.0119 & 0.7270 & 0.0594 & 0.0233 & 0.0305 \\
\hline 0.0 & 0.4 & -0.0182 & 0.6871 & 0.0630 & 0.0277 & 0.0345 \\
\hline 0.0 & 0.5 & -0.0663 & 0.6297 & 0.0697 & 0.0344 & 0.0400 \\
\hline 0.0 & 0.6 & -0.1417 & 0.5441 & 0.0821 & 0.0447 & 0.0475 \\
\hline 0.0 & 0.7 & -0.2774 & 0.4034 & 0.1046 & 0.0618 & 0.0591 \\
\hline 0.0 & 0.8 & -0.5699 & 0.1386 & 0.1572 & 0.0969 & 0.0796 \\
\hline 0.0 & 0.9 & -1.4789 & 0.5639 & 0.3438 & 0.2021 & 0.1310 \\
\hline 0.1 & 0.0 & 0.0598 & 0.9320 & 0.0587 & 0.0111 & 0.0365 \\
\hline 0. & 0.1 & 0.0462 & 0.9165 & 0.0585 & 0.0114 & 0.0390 \\
\hline 0.1 & 0.2 & 0.0375 & 0.9040 & 0.0594 & 0.0132 & 0.0432 \\
\hline 0.1 & 0.3 & 0.0267 & 0.8896 & 0.0602 & 0.0161 & 0.0496 \\
\hline 0.1 & 0.4 & 0.0134 & 0.8666 & 0.0632 & 0.0213 & 0.0596 \\
\hline 0.1 & 0.5 & -0.0040 & 0.8366 & 0.0681 & 0.0301 & 0.0744 \\
\hline 0.1 & 0.6 & -0.0433 & 0.7790 & 0.0796 & 0.0464 & 0.0978 \\
\hline 0.1 & 0.7 & & 0.6722 & 0.1049 & 0.0785 & 0.1395 \\
\hline 0.1 & 0.8 & -0.3634 & 0.3700 & 0.1671 & 0.1430 & 0.2046 \\
\hline 0.2 & 0.0 & 0.0668 & 0.9414 & 0.0649 & 0.0043 & 0.0445 \\
\hline 0.2 & 0.1 & 0.0563 & 0.9227 & 0.0648 & 0.0042 & 0.0483 \\
\hline $0 . \overline{2}$ & $0 . \overline{2}$ & 0.0482 & 0.9104 & 0.0649 & 0.0053 & 0.0551 \\
\hline $0 . \overline{2}$ & $0 . \overline{3}$ & 0.0399 & 0.8922 & 0.0648 & 0.0076 & 0.0659 \\
\hline 0.2 & 0.4 & 0.0277 & 0.8650 & 0.0658 & 0.0123 & 0.0813 \\
\hline 0.2 & 0.5 & 0.0103 & 0.8228 & 0.0698 & 0.0218 & 0.1073 \\
\hline 0.2 & 0.6 & -0.0292 & 0.7336 & 0.0793 & 0.0424 & 0.1536 \\
\hline 0.2 & 0.7 & -0.1515 & 0.4679 & 0.1021 & 0.0824 & 0.2190 \\
\hline $0 . \overline{3}$ & 0.0 & 0.0822 & 0.9334 & 0.0753 & -0.0054 & 0.0528 \\
\hline 0.3 & 0.1 & 0.0735 & 0.9130 & 0.0753 & -0.0066 & 0.0586 \\
\hline 0.3 & 0.2 & 0.0651 & 0.8953 & 0.0750 & -0.0071 & 0.0695 \\
\hline 0.3 & 0.3 & 0.0608 & 0.8731 & 0.0747 & -0.0065 & 0.0857 \\
\hline 0.3 & 0.4 & 0.0521 & 0.8321 & 0.0752 & -0.0031 & 0.1124 \\
\hline 0.3 & 0.5 & 0.0386 & 0.7488 & 0.0755 & 0.0030 & 0.1592 \\
\hline 0.3 & 0.6 & -0.0070 & 0.4951 & 0.0812 & 0.0223 & 0.2284 \\
\hline 0.4 & 0.0 & 0.1072 & 0.9207 & 0.0950 & -0.0194 & 0.0640 \\
\hline 0.4 & 0.1 & 0.1007 & 0.8932 & 0.0959 & -0.0232 & 0.0727 \\
\hline 0.4 & 0.2 & 0.0992 & 0.8707 & 0.0965 & -0.0274 & 0.089 \\
\hline 0.4 & 0.3 & 0.0983 & 0.8309 & 0.0970 & -0.0323 & 0.1165 \\
\hline 0.4 & 0.4 & 0.1044 & 0.7499 & 0.0974 & -0.0379 & 0.1665 \\
\hline 0.4 & 0.5 & 0. & 0.5000 & 0.0966 & -0.0382 & 0.2301 \\
\hline $0 . \underline{5}$ & 0.0 & 0 . & 983 & 0.1 & -0.0416 & 0.0777 \\
\hline 0.5 & 0.1 & 19 & 632 & 0.1 & -0.0511 & 0.0928 \\
\hline 0 & 0.2 & 0.1606 & 0.8220 & 0.14 & -0.0629 & 0 . \\
\hline 0.5 & 0.3 & 70 & 0.7397 & 0.14 & -0.0820 & 0 \\
\hline 0.5 & 0.4 & 37 & 0.4932 & 0.1527 & -0.1021 & 0.2321 \\
\hline 0.6 & 0.0 & 0.2186 & 0.8658 & 0.2001 & -0.0784 & 0.0978 \\
\hline 0.6 & 0.1 & 0.2441 & & 0.2165 & -0.0984 & 0.1219 \\
\hline 0.6 & 0.2 & 76 & 292 & & -0.1315 & 0.1680 \\
\hline 0.6 & $0 . \overline{3}$ & 0.4476 & $0.4 \overline{8} \overline{2}$ & $0 . \overline{2} 642$ & -0.1749 & 0.2367 \\
\hline 0.7 & 0.0 & 0.3461 & 0.8151 & 0.3520 & -0.1456 & 0.1290 \\
\hline 0.7 & 0.1 & 0.4318 & 0.7154 & 0.4077 & -0.1955 & 0.1723 \\
\hline 0.7 & 0.2 & 0.6762 & 0.4657 & 0.4 & -0.2590 & \\
\hline 0.8 & 0.0 & 0.6516 & 0.7079 & 0.7220 & -0.2739 & 0.1750 \\
\hline 0 & 01 & 0.8226 & 80 & 0.7 & -0.3705 & \\
\hline 0.9 & 0.0 & 0.9679 & 0.5222 & 0.8683 & -0.4520 & 0.2360 \\
\hline
\end{tabular}


Table 3.7: Means, variances and covariances of the proposed estimators of parameters of type I (maximum) extreme value distribution $(\theta=0, \sigma=1)$ from 100000 samples of size $n=24$.

\begin{tabular}{cc|ccccc}
\hline$p_{1}$ & $p_{2}$ & $M(\hat{\theta})$ & $M(\hat{\sigma})$ & $V(\hat{\theta})$ & $C o v(\hat{\theta}, \hat{\sigma})$ & $V(\hat{\sigma})$ \\
\hline 0.0 & 0.0 & 0.0599 & 0.8235 & 0.0461 & 0.0148 & 0.0213 \\
0.0 & 0.1 & 0.0101 & 0.7468 & 0.0447 & 0.0132 & 0.0193 \\
0.0 & 0.2 & 0.0163 & 0.7655 & 0.0466 & 0.0160 & 0.0228 \\
0.0 & 0.3 & -0.0378 & 0.6936 & 0.0462 & 0.0158 & 0.0226 \\
0.0 & 0.4 & -0.0437 & 0.6893 & 0.0503 & 0.0206 & 0.0274 \\
0.0 & 0.5 & -0.0606 & 0.6673 & 0.0579 & 0.0280 & 0.0342 \\
0.0 & 0.6 & -0.1718 & 0.5419 & 0.0625 & 0.0324 & 0.0370 \\
0.0 & 0.7 & -0.2673 & 0.4449 & 0.0844 & 0.0499 & 0.0500 \\
0.0 & 0.8 & -0.6445 & 0.0760 & 0.1203 & 0.0744 & 0.0621 \\
0.0 & 0.9 & -1.5614 & -0.6349 & 0.2817 & 0.1671 & 0.1088 \\
0.5 & 0.0 & -1.2024 & 2.3564 & 0.1415 & -0.2313 & 0.4098 \\
0.5 & 0.1 & 0.1988 & 0.7197 & 0.1116 & -0.0394 & 0.0619 \\
0.5 & 0.2 & 0.1548 & 0.7928 & 0.1155 & -0.0523 & 0.0956 \\
0.5 & 0.3 & 0.2438 & 0.5225 & 0.1209 & -0.0635 & 0.1055 \\
0.5 & 0.4 & 0.3035 & 0.3614 & 0.1229 & -0.0764 & 0.1550 \\
\hline
\end{tabular}

Table 3.8: Means, variances and covariances of the proposed estimators of parameters of type I (maximum) extreme value distribution $(\theta=0, \sigma=1)$ from 100000 samples of size $n=28$.

\begin{tabular}{cc|ccccc}
\hline$p_{1}$ & $p_{2}$ & $M(\hat{\theta})$ & $M(\hat{\sigma})$ & $V(\hat{\theta})$ & $\operatorname{Cov}(\hat{\theta}, \hat{\sigma})$ & $V(\hat{\sigma})$ \\
\hline 0.0 & 0.0 & 0.0521 & 0.8403 & 0.0398 & 0.0124 & 0.0185 \\
0.0 & 0.1 & 0.0271 & 0.8043 & 0.0394 & 0.0122 & 0.0185 \\
0.0 & 0.2 & 0.0039 & 0.7725 & 0.0397 & 0.0129 & 0.0193 \\
0.0 & 0.3 & -0.0238 & 0.7375 & 0.0401 & 0.0141 & 0.0208 \\
0.0 & 0.4 & -0.0598 & 0.6937 & 0.0418 & 0.0163 & 0.0231 \\
0.0 & 0.5 & -0.0559 & 0.6981 & 0.0496 & 0.0236 & 0.0299 \\
0.0 & 0.6 & -0.1292 & 0.6137 & 0.0561 & 0.0299 & 0.0349 \\
0.0 & 0.7 & -0.2601 & 0.4773 & 0.0702 & 0.0413 & 0.0431 \\
0.0 & 0.8 & -0.5492 & 0.2011 & 0.1042 & 0.0652 & 0.0572 \\
0.0 & 0.9 & -1.6473 & -0.7132 & 0.2474 & 0.1482 & 0.0965 \\
0.5 & 0.0 & -1.2435 & 2.4089 & 0.1344 & -0.2161 & 0.3754 \\
0.5 & 0.1 & 0.1291 & 0.8530 & 0.0948 & -0.0365 & 0.0648 \\
0.5 & 0.2 & 0.1529 & 0.7728 & 0.0998 & -0.0458 & 0.0801 \\
0.5 & 0.3 & 0.1931 & 0.6407 & 0.1039 & -0.0577 & 0.1052 \\
0.5 & 0.4 & 0.3187 & 0.2749 & 0.1028 & -0.0619 & 0.1152 \\
\hline
\end{tabular}


Table 3.9: Means, variances and covariances of the proposed estimators of parameters of type I (maximum) extreme value distribution $(\theta=0, \sigma=1)$ from 100000 samples of size $n=30$.

\begin{tabular}{|c|c|c|c|c|c|c|}
\hline$p_{1}$ & $p_{2}$ & $M(\hat{\theta})$ & $M(\hat{\sigma})$ & $V(\hat{\theta})$ & $\operatorname{Cov}(\hat{\theta}, \hat{\sigma})$ & $V(\hat{\sigma})$ \\
\hline 0.0 & 0.0 & 0.0492 & 0.8472 & 0.0371 & 0.0114 & 0.0175 \\
\hline 0.0 & 0.1 & 0.0366 & 0.8289 & 0.0372 & 0.0120 & 0.0182 \\
\hline 0.0 & 0.2 & 0.0222 & 0.8101 & 0.0379 & 0.0131 & 0.0195 \\
\hline 0.0 & 0.4 & -0.0165 & 0.7567 & 0.0419 & 0.0176 & 0.0242 \\
\hline & & & 111 & 0.0464 & 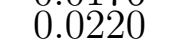 & 0.028 \\
\hline 0.0 & 0.6 & -0.1107 & 0.6446 & 0.0537 & & 0.0340 \\
\hline 0.0 & 0.7 & -0.2165 & 0.5353 & 0.0687 & 0.0410 & 0.0433 \\
\hline (0.) & & 488 & 0.3232 & 0.1026 & 650 & 0.0589 \\
\hline 0 . & 0.9 & 682 & -0.2377 & 0.2205 & & \\
\hline & 0.0 & -0.7213 & 1.9458 & 0.0850 & -0.1039 & 0.2392 \\
\hline & & 0.0314 & 0.9435 & 0.0393 & 0.0073 & 0.0266 \\
\hline 0 & 0.2 & 0.0253 & 0.9339 & 0.0392 & 0.0082 & 0.0295 \\
\hline & & 0.0175 & 0.9253 & 0.0401 & 105 & 0.0345 \\
\hline & 0.4 & 0.1 & 0.9099 & 0.0417 & & 0.04 \\
\hline & 0.5 & -0.0047 & 0.8876 & 0.0455 & 0.0202 & 0.0520 \\
\hline & & .0287 & 0.8503 & 0.0528 & 0.0315 & 0.0693 \\
\hline & 0.7 & -0.0 & 0.7743 & & & 0.1026 \\
\hline & 0.8 & -0.2459 & 0.5731 & 0.1259 & 0.1209 & 0.1812 \\
\hline & & 57038 & & & & 0.2618 \\
\hline U. & 0.1 & 0.0360 & 0.9469 & 0.0427 & 0.0026 & 0.0326 \\
\hline & 0.2 & 0.0310 & 0.9390 & 0.0426 & 0.0032 & 0.0378 \\
\hline & & & 0.9 & & & 0.0 \\
\hline 0. & 0.4 & 0.019 & 0.9109 & 0.0439 & & 0.0570 \\
\hline & 0.5 & 0.0068 & 0.8803 & 0.0459 & 48 & 0.0761 \\
\hline & 0.6 & -0.0199 & 0.8169 & 0.0524 & & 011 \\
\hline 0 . & 0.7 & -0.1004 & 0.6433 & 0.0726 & 05 & 0.1965 \\
\hline & & & & & & 0.28 \\
\hline & 0. & 0.0483 & & 0.0493 & & 0.0 \\
\hline 0.3 & 0.2 & 0.0426 & 0.9300 & 0.0491 & -0 . & 0.0479 \\
\hline & & & & & & \\
\hline 0 & 0.4 & 0.0342 & & 0.0492 & 32 & 0.0 \\
\hline & 0.5 & 0.0256 & 44 & 0.0501 & 0.0021 & 0.1182 \\
\hline & & & & & & \\
\hline 0.4 & 0 . & & & 0.1 & 43 & 0.3229 \\
\hline & 0 & 0.0662 & 0.9301 & 0.0 & 160 & 0.0508 \\
\hline & 0.2 & & & 38 & & \\
\hline 0.4 & 0. & 0 . & & 5 & & 0.08 \\
\hline & 0.4 & & & & & 0.1201 \\
\hline & 0.5 & 0.0853 & 0.6 & 0.06 & 344 & 0.2045 \\
\hline 0. & 0.0 & -1.2641 & & & & \\
\hline & & & 0.9072 & & & 0.0643 \\
\hline 0 . & 0. & 0. & 0.88 & 0.0928 & 443 & 0.0850 \\
\hline & 0.3 & 0.123 & 0.8279 & 0.0995 & -0 . & 0.1232 \\
\hline & 0.4 & & & 2 & & \\
\hline $0 .(\mathrm{r}-\mathrm{x}$ & 08 & 94 & & 6 & & 0.4291 \\
\hline & 0. & 0.1639 & & 0.1441 & -0.0696 & 0.086 \\
\hline & & & & & & \\
\hline & 0.3 & 0.2972 & & 0.2 & -0.1 & 0.2 \\
\hline & 0.0 & -2.6295 & & 0.4029 & 4618 & .5298 \\
\hline & 0.1 & 0.2894 & & 0.2835 & -0.1 & $\begin{array}{l}0.1279 \\
0.2008\end{array}$ \\
\hline (1 & 0.0 & $\begin{array}{r}0.4512 \\
-4.1412\end{array}$ & $\begin{array}{l}0.6423 \\
3.4443\end{array}$ & $\begin{array}{l}0.3701 \\
0.9823\end{array}$ & $\begin{array}{l}95 \\
06\end{array}$ & 0.7244 \\
\hline & 0. & 0.6786 & 0.62 & 0.692 & -0.3259 & 0.2092 \\
\hline 0.0 & 0.0 & -7.4112 & 4.1440 & 3.6210 & -2.0831 & 1.2069 \\
\hline
\end{tabular}




\section{Chapter 4}

\section{Estimation of $\theta$ in the model $\frac{1}{c \theta} f\left(\frac{x-\theta}{c \theta}\right)$ on the basis of complete and censored samples}

Using the asymptotic covariance matrix of the estimators for the extreme value distribution we now construct an efficient estimator of $\theta$ in the reduced model $\frac{1}{c \theta} f\left(\frac{x-\theta}{c \theta}\right)$ where $\theta(>0)$ is unknown and $c(>0)$ is known for complete and censored samples.

We construct an estimator

$$
T_{n}=n^{-1} \sum_{j=1}^{n} J\left(\frac{j}{n+1}\right) X_{j n}
$$

of $\theta(\theta>0)$ in the pdf $\frac{1}{c \theta} f\left(\frac{x-\theta}{c \theta}\right)$ where $c(>0)$ is known and $J$ is a well behaved weight function. The estimator $T_{n}$ is asymptotically normally distributed with mean $\theta$ and variance $\theta^{2}(n I)^{-1}$ where $I$ is the Fisher information. Then we obtain a similar estimator $T_{n}^{*}$ of the form $T_{n}$ on the basis of a type II censored sample where 
additional weights are given to certain sample percentiles. Again, the estimator $T_{n}^{*}$ is asymptotically normally distributed with mean $\theta$ and variance $\theta^{2}\left(n I^{*}\right)^{-1}$ where $I^{*}$ is the Fisher information for the corresponding problem.

\subsection{Complete sample}

Using the notations in Chapter 2, the Fisher information is given by $\theta^{-2} I$ where

$$
\begin{gathered}
I=\operatorname{Var}\{L(Y)\}, \quad Y=\frac{X_{1}-\theta}{c \theta} \\
\text { and } L(y)=-\left\{1+\left(y+c^{-1}\right) \frac{f^{\prime}(y)}{f(y)}\right\}=c^{-1} L_{1}(y)+L_{2}(y)
\end{gathered}
$$

(Samanta (1985)).

Clearly,

$$
\begin{aligned}
I & =E\left\{L^{2}(Y)\right\} \\
& =E\left\{c^{-2} L_{1}^{2}(y)+L_{2}^{2}(y)+2 c^{-1} L_{1}(y) L_{2}(y)\right\} \\
& =E\left\{c^{-2} L_{1}^{2}(y)\right\}+E\left\{L_{2}^{2}(y)\right\}+E\left\{2 c^{-1} L_{1}(y) L_{2}(y)\right\} \\
& =c^{-2}+2 c^{-1}(\gamma-1)+\frac{\pi^{2}}{6}+(\gamma-1)^{2}
\end{aligned}
$$

We construct an estimator $T_{n}$ of $\theta$ defined by

$$
T_{n}=n^{-1} \sum_{j=1}^{n} J\left(\frac{j}{n+1}\right) X_{j n}
$$


where

$$
J(u)=(c I)^{-1} L^{\prime}(-\ln (-\ln u))
$$

We have, $L(y)=c^{-1} L_{1}(y)+L_{2}(y)$

$$
\begin{aligned}
L^{\prime}(y) & =c^{-1} L_{1}^{\prime}(y)+L_{2}^{\prime}(y) \\
& =c^{-1} e^{-y}+1+y e^{-y}-e^{-y} \\
L^{\prime}(\ln (-\ln u))= & c^{-1} e^{\ln (-\ln u)}+1-\ln (-\ln u) e^{\ln (-\ln u)}-e^{\ln (-\ln u)} \\
= & -c^{-1} \ln u+1+\ln (-\ln u) \ln u+\ln u \\
= & 1+\ln u\left[1-c^{-1}+\ln (-\ln u)\right] \\
J(u)= & (c I)^{-1} L^{\prime}(-\ln (-\ln u)) \\
= & {\left[c^{-1}+2(\gamma-1)+c\left\{\frac{\pi^{2}}{6}+(\gamma-1)^{2}\right\}\right]^{-1}\left[1+\ln u\left\{1-c^{-1}+\ln (-\ln u)\right\}\right], } \\
& \quad 0<u<1 .
\end{aligned}
$$

It follows from the results in Chernoff et. al. (1967) that $T_{n}$ is asymptotically normally distributed with mean $\theta$ and variance $\theta^{2}(n I)^{-1}$.

\subsection{Censored sample}

We assume type II censoring as in chapter 3 and use the notations introduced in 
this chapter. The Fisher information for this problem is $\theta^{-2} I^{*}$ where

$$
\begin{aligned}
I^{*}= & \int_{\lambda_{1}}^{\lambda_{2}} L^{2}(y) f(y) d y+\frac{f^{2}\left(\lambda_{1}\right)\left(\lambda_{1}+c^{-1}\right)^{2}}{p_{1}}+\frac{f^{2}\left(\lambda_{2}\right)\left(\lambda_{2}+c^{-1}\right)^{2}}{p_{2}} \\
= & \int_{\lambda_{1}}^{\lambda_{2}}\left\{c^{-1} L_{1}(y)+L_{2}(y)\right\}^{2} f(y) d y+\frac{f^{2}\left(\lambda_{1}\right)\left(\lambda_{1}^{2}+2 \lambda_{1} c^{-1}+c^{-2}\right)}{p_{1}} \\
& +\frac{f^{2}\left(\lambda_{2}\right)\left(\lambda_{2}^{2}+2 \lambda_{2} c^{-1}+c^{-2}\right)}{p_{2}} \\
= & \int_{\lambda_{1}}^{\lambda_{2}} c^{-2} L_{1}^{2}(y) f(y) d y+2 \int_{\lambda_{1}}^{\lambda_{2}} c^{-1} L_{1}(y) L_{2}(y) f(y) d y+\int_{\lambda_{1}}^{\lambda_{2}} L_{2}^{2}(y) f(y) d y \\
& +\frac{f^{2}\left(\lambda_{1}\right) \lambda_{1}^{2}}{p_{1}}+\frac{2 f^{2}\left(\lambda_{1}\right) \lambda_{1} c^{-1}}{p_{1}}+\frac{f^{2}\left(\lambda_{1}\right) c^{-2}}{p_{1}}+\frac{f^{2}\left(\lambda_{2}\right) \lambda_{2}^{2}}{p_{2}}+\frac{2 f^{2}\left(\lambda_{2}\right) \lambda_{2} c^{-1}}{p_{2}}+\frac{f^{2}\left(\lambda_{2}\right) c^{-2}}{p_{2}} \\
= & c^{-2} \mathcal{I}_{11}^{*}+2 c^{-1} \mathcal{I}_{12}^{*}+\mathcal{I}_{22}^{*}[\text { using equation }(3.1),(3.2),(3.3)]
\end{aligned}
$$

The proposed estimator $T_{n}^{*}$ of $\theta$ is

$$
T_{n}^{*}=n^{-1} \sum_{j=s}^{l} J^{*}\left(\frac{j}{n+1}\right) X_{j n}+a X_{s n}+b X_{l n}
$$


where

$$
\begin{aligned}
& J^{*}(u)=\left(c I^{*}\right)^{-1}\left[1+\ln u\left\{1-c^{-1}+\ln (-\ln u)\right\}\right], \quad p_{1}<u<1-p_{2} \\
& a=\left(c I^{*}\right)^{-1} f\left(\lambda_{1}\right)\left[L\left(\lambda_{1}\right)+\frac{f\left(\lambda_{1}\right)\left(\lambda_{1}+c^{-1}\right)}{p_{1}}\right] \\
& =\left(c \mathcal{I}_{22}^{*}+2 \mathcal{I}_{12}^{*}+c^{-1} \mathcal{I}_{11}^{*}\right)^{-1} e^{-\lambda_{1}-e^{-\lambda_{1}}} \\
& {\left[c^{-1}-c^{-1} e^{-\lambda_{1}}-1+\lambda_{1}-\lambda_{1} e^{-\lambda_{1}}+\frac{e^{-\lambda_{1}-e^{-\lambda_{1}}}\left(\lambda_{1}+c^{-1}\right)}{p_{1}}\right]} \\
& =\left(c \mathcal{I}_{22}^{*}+2 \mathcal{I}_{12}^{*}+c^{-1} \mathcal{I}_{11}^{*}\right)^{-1} e^{r_{1}-e^{r_{1}}} \\
& {\left[c^{-1}-c^{-1} e^{r_{1}}-1-r_{1}+r_{1} e^{r_{1}}+\frac{e^{-r_{1}-e^{-r_{1}}}\left(-r_{1}+c^{-1}\right)}{p_{1}}\right]} \\
& =\left(c \mathcal{I}_{22}^{*}+2 \mathcal{I}_{12}^{*}+c^{-1} \mathcal{I}_{11}^{*}\right)^{-1} e^{\ln \left(-q_{1}\right)-e^{\ln \left(-q_{1}\right)}} \\
& {\left[c^{-1}-c^{-1} e^{\ln \left(-q_{1}\right)}-1-r_{1}+r_{1} e^{\ln \left(-q_{1}\right)}+\frac{e^{\ln \left(-q_{1}\right)-e^{\ln \left(-q_{1}\right)}\left(-r_{1}+c^{-1}\right)}}{p_{1}}\right]} \\
& =\left(c \mathcal{I}_{22}^{*}+2 \mathcal{I}_{12}^{*}+c^{-1} \mathcal{I}_{11}^{*}\right)^{-1}\left(-p_{1} q_{1}\right)\left[c^{-1}+c^{-1} q_{1}-1-r_{1}-r_{1} q_{1}+\frac{-p_{1} q_{1}\left(-r_{1}+c^{-1}\right)}{p_{1}}\right] \\
& =\left(c \mathcal{I}_{22}^{*}+2 \mathcal{I}_{12}^{*}+c^{-1} \mathcal{I}_{11}^{*}\right)^{-1}\left(-p_{1} q_{1}\right)\left[c^{-1}+c^{-1} q_{1}-1-r_{1}-r_{1} q_{1}-q_{1}\left(-r_{1}+c^{-1}\right)\right] \\
& =\left(c \mathcal{I}_{22}^{*}+2 \mathcal{I}_{12}^{*}+c^{-1} \mathcal{I}_{11}^{*}\right)^{-1} p_{1} q_{1}\left(1+r_{1}-c^{-1}\right),
\end{aligned}
$$




$$
\begin{aligned}
& b=\left(c I^{*}\right)^{-1} f\left(\lambda_{2}\right)\left[-L\left(\lambda_{2}\right)+\frac{f\left(\lambda_{2}\right)\left(\lambda_{2}+c^{-1}\right)}{p_{2}}\right] \\
& =\left(c \mathcal{I}_{22}^{*}+2 \mathcal{I}_{12}^{*}+c^{-1} \mathcal{I}_{11}^{*}\right)^{-1} e^{-\lambda_{2}-e^{-\lambda_{2}}} \\
& {\left[-c^{-1}+c^{-1} e^{-\lambda_{2}}+1-\lambda_{2}+\lambda_{2} e^{-\lambda_{2}}+\frac{e^{-\lambda_{2}-e^{-\lambda_{2}}}\left(\lambda_{2}+c^{-1}\right)}{p_{2}}\right]} \\
& =\left(c \mathcal{I}_{22}^{*}+2 \mathcal{I}_{12}^{*}+c^{-1} \mathcal{I}_{11}^{*}\right)^{-1} e^{r_{2}-e^{r_{2}}} \\
& {\left[-c^{-1}+c^{-1} e^{r_{2}}+1+r_{2}-r_{2} e^{r_{2}}+\frac{e^{r_{2}-e^{r_{2}}}\left(-r_{2}+c^{-1}\right)}{p_{2}}\right]} \\
& =\left(c \mathcal{I}_{22}^{*}+2 \mathcal{I}_{12}^{*}+c^{-1} \mathcal{I}_{11}^{*}\right)^{-1} e^{\ln \left(-q_{2}\right)-e^{\ln \left(-q_{2}\right)}} \\
& {\left[-c^{-1}+c^{-1} e^{\ln \left(-q_{2}\right)}+1+r_{2}-r_{2} e^{\ln \left(-q_{2}\right)}+\frac{e^{-\ln \left(-q_{2}\right)-e^{\ln \left(-q_{2}\right)}\left(-r_{2}+c^{-1}\right)}}{p_{2}}\right]} \\
& =\left(c \mathcal{I}_{22}^{*}+2 \mathcal{I}_{12}^{*}+c^{-1} \mathcal{I}_{11}^{*}\right)^{-1}(-)\left(1-p_{2}\right) q_{2} \\
& {\left[-c^{-1}-c^{-1} q_{2}+1+r_{2}+r_{2} q_{2}+\frac{-\left(1-p_{2}\right) q_{2}\left(-r_{2}+c^{-1}\right)}{p_{2}}\right]} \\
& =\left(c \mathcal{I}_{22}^{*}+2 \mathcal{I}_{12}^{*}+c^{-1} \mathcal{I}_{11}^{*}\right)^{-1}(-)\left(1-p_{2}\right) q_{2} \\
& {\left[-c^{-1}-c^{-1} q_{2}+1+r_{2}+r_{2} q_{2}-\frac{q_{2}\left(-r_{2}+c^{-1}\right)}{p_{2}}+q_{2}\left(-r_{2}+c^{-1}\right)\right]} \\
& =\left(c \mathcal{I}_{22}^{*}+2 \mathcal{I}_{12}^{*}+c^{-1} \mathcal{I}_{11}^{*}\right)^{-1}(-)\left(1-p_{2}\right) q_{2} \\
& {\left[-c^{-1}-c^{-1} q_{2}+1+r_{2}+r_{2} q_{2}-\frac{q_{2}\left(-r_{2}+c^{-1}\right)}{p_{2}}+q_{2}\left(-r_{2}+c^{-1}\right)\right]} \\
& \left.=\left(c \mathcal{I}_{22}^{*}+2 \mathcal{I}_{12}^{*}+c^{-1} \mathcal{I}_{11}^{*}\right)^{-1}(-)\left(1-p_{2}\right) q_{2}\right)\left[-c^{-1}+1+r_{2}-\frac{q_{2}}{p_{2}}\left(-r_{2}+c^{-1}\right)\right] \\
& =\left(c \mathcal{I}_{22}^{*}+2 \mathcal{I}_{12}^{*}+c^{-1} \mathcal{I}_{11}^{*}\right)^{-1}\left(1-p_{2}\right) q_{2}\left[c^{-1}-r_{2}-1+\frac{q_{2}\left(c^{-1}-r_{2}\right)}{p_{2}}\right] .
\end{aligned}
$$


The estimator $T_{n}^{*}$ is asymptotically normally distributed with mean $\theta$ and variance $\theta^{2}\left(n I^{*}\right)^{-1}$

We show the necessary calculations for the estimator $T_{n}^{*}$ in two special cases.

Case 1. With $p_{1}=p_{2}=0.05$ and $c=1$ we have

$$
\begin{aligned}
I^{*} & =1.8274108, \quad J^{*}(u)=0.5472+0.5472 \ln u[\ln (-\ln u)], \quad 0.05<u<0.95 \\
a & =-0.0899, \quad b=0.0294 .
\end{aligned}
$$

Case 2. With $p_{1}=p_{2}=0.1$ and $c=1$ we have

$$
\begin{aligned}
I^{*} & =1.7164408, \quad J^{*}(u)=0.5826+0.5826 \ln u[\ln (-\ln u)], \quad 0.1<u<0.9 \\
a & =-0.1119, \quad b=0.0649 .
\end{aligned}
$$




\section{Chapter 5}

\section{Conclusions}

New estimators of the parameters of type-I extreme value distribution have been proposed in the thesis. We have provided explicit mathematical expressions for the efficient estimators of the parameters without solving the likelihood equations for both complete and censored samples. Our estimators are linear combinations of order statistics where the coefficients of these linear combinations are smooth weight functions given in Chernoff et. al. (1967). For both complete and censored cases weight functions are well behaved and have explicit formulas. However, additional weights are given to certain sample percentiles for censored samples. Our estimators are asymptotically normally distributed and their variances attain the Cramer-Rao Lower bound. We have also derived the explicit formulas for the appropriate information matrices. We obtain simple expressions for the elements of Fisher Information matrices even in the censored cases. We also construct efficient estimators in a reduced model for complete and censored samples. It follows that the estimator is asymptotically normally distributed.

We have also performed an extensive Monte Carlo simulation study to find the means, variances and covariances of the estimators from 100000 samples for sample 
sizes $n=10,16,20,24$ and $n=30$ for different degrees of censoring. The study shows that the bias of the estimators increases as the degree of censoring increases, and the bias decreases as the sample size increases. As expected, the variances of the estimators decrease as the sample size increases.

The estimators proposed in this thesis have several advantages compared to other existing methods of estimation. We have provided quite flexible methods to compute these estimators both for complete and censored samples. We have also given for the first time the explicit formulas for the appropriate information matrix. Our estimators perform reasonably well and compares favourably with the estimators of Harter and Moore (1968). Harter and Moore (1968) developed the method of estimation of the parameters of extreme value distribution by solving the likelihood equations. These equations have very complicated expressions and are extremely difficult to solve. Harter and Moore (1968) suggested iteration techniques and an appropriate computer program to solve these equations.

In summary, this thesis obtains efficient estimators of the parameters of the extreme value distribution 'without' sloving the likelihood equations, and provides for the 'first' time simple expressions for the elements of the information matrix for type II censoring. These works are of course useful additions to the current statistical literature. This research provides novel and useful methods for efficient estimation of parameters, and in particular, the methodologies can be applied in many practical situations where the extreme value distribution is used as an appropriate statistical model. This research will provide efficient and practical tools to applied researchers in meteorology, quality control, population studies, economics, naval engineering, astronomy, oceanography and many other areas. 
We would like to apply our methodologies in some real data sets for which the extreme value distribution is of interest. Future study will cover these applications. 


\section{Bibliography}

Abramowitz, M. and I. A. Stegun (1970). Handbook of Mathematical Functions with Formulas, Graphs and Mathematical Tables. Dover Publications, INC, New York.

Balakrishnan, N. and P. Chan (1992a). Order statistics from extreme value distribution, ii: best linear unbised estimates and other uses. Communications in Statistics-Simulation and Computation 21(4), 1219-1246.

Balakrishnan, N. and P. Chan (1992b). Order statistics from extreme value distribution, i:table of means, variances and covariances. Communications in StatisticsSimulation and Computation 21(4), 1199-1217.

Balakrishnan, N., N. Kannaa, C. T. Lin, and S. J. S. Wu (2004). Inference for the extreme value distribution under progressive type-ii censoring. Journal of Statistical Computation and Simulation 74(1), 25-45.

Balcerak, E. (2011). Estimating the distribution of extreme events. Research Spotlight 92(28), 240-241.

Bartoszynski, R. and M. Niewiadomska-Bugaj (2009). Probability and Statistical Inference. Wiley-Interscience. 
Bennett, C. (1952). Asymptotic properties of ideal linear estimators. Ph. D. thesis, University of Michigan.

Bhoj, D. (1997). Estimation of parameters of the extreme value distribution using ranked set sampling. Communications in Statistics-Theory and Methods 26(3), 653-667.

Bickel, P. (1973). On some analogues to linear combinations of order statistics in the linear model. Annals of Statistics 1, 597-616.

Broussard, P. J. (1998). The behavior of extreme values in germany's stock index futures: An application to intradaily margin setting. European Journal of Operational Research 1(104), 393-402.

Casella, G. and R. Berger (2002). Statistical Inference. Duxbury, Thomson Learning.

Chen, Z. (1998). Joint estimation for the parameters of the extreme value distributions. Statistical Papers 1(39), 135-146.

Chernoff, H., J. L. Gastwirth, and J. Johns, M. V. (1967). Asymptotic distribution of linear combinations of functions of order statistics with applications to estimation. Annals of Mathematical Statistics 1(38), 52-72.

Chernoff, H. and I. Savage (1958). Asymptotic normality and efficiency of certain nonparametric test statistics. Annals of Mathematical Statistics 29, 972-994.

Daniell, P. (1920). Observations weighted according to order. American Journal of Mathematics 42, 222-236. 
David., H. A. and H. N. Nagaraja (2003). Order Statistics, Third Edition. John Wiley \& Sons, Hoboken. New Jersey.

Deheuvels, P. and T. Oliveira (1989). On the non-parametric estimation of the bivariate extreme-value distributions. Statistics $\&$ Probability Letters 8, 315-323.

Downton, F. (1966, Feb). Linear estimates of parameters in the extreme value distribution. Technometrics 8(1), 3-17.

Einmahl, J., L. Haan, and X. Huang (1993). Estimating a multidimensional extreme value distribution. Journal of Multivariate Analysis 47, 35-47.

Fard, P. and B. Holmquist (2007). First moment approximations for order statistics from the extreme value distribution. Statistical Methodology 4, 196-203.

Fard, P. and B. Holmquist (2008). Approximations of variances and covariances for order statistics from the standard extreme value distribution. Communications in Statistics-Simulation and Computation 37(8), 1500-1506.

Galambos, J. (1978). The asymptotic theory of extreme order Statistics. John Wiley, New York.

Haan, L. and A. Rerreira (2006). Extreme Value Theory. Springer.

Haan, L. and S. Resnick (1993). Estimating the limit distribution of multivariate extremes. Communications in Statistics. Stochastic Models 9(2), 275-309.

Harter, H. L. and A. H. Moore (1968). Maximum-likelihood estimation, from doubly censored samples, of the parameters of the first asymptotic distribution of extreme values. Journal of the American Statistical Association 323(63), 889-901. 
Hassanein, K. (1968). Analysis of extreme value data by sample quantiles for very large samples. Journal of the American Statistical Association 63, 877-888.

Hassanein, K. (1969). Estimation of the parameters of the extreme value distribution by use of two or three order statistics. Biometrika 56(2), 429-436.

Hassanein, K. (1972). Simultaneous estimation of the parameters of the extreme value distribution by sample quantiles. Technometrics 14(1), 63-70.

Hosking, J. R. M. (1984). Testing whether the shape parameter is zero in the generalized extreme value distribution. Biometrika 71 (2), 367-374.

Hosking, J. R. M., J. R. Wallis, and E. F. Wood (1985). Estimation of the generalized extreme value distribution by the method of probability-weighted moments. Technometrics 27(3), 251-261.

Johnson, N. L., S. Kotz, and N. Balakrishnan (1995). Continuous Univariate Distributions. Second Edition, John Wiley \& Sons, New York.

Kimball, B. (1956). The bias in certain estimates of the parameters of the extreme value distribution. The Annals of Mathematical Statistics 27(3), 758-767.

Kotz, S. and S. Nadarajah (2000). Extreme Value Distrributions Theory and Applications. Imperial College Press, London.

Koutsoyiannis, D. (2004). Statistics of extreme and estimation of extreme rainfall: Theoretical investigation. Hydrological Sciences 49(4), 575-590.

Lawless, F. J. (2003). Statistical Models and Methods for Lifetime Data. John Wiley \& Sons, New Jersey. 
Lieblein, J. (1953). On the exact evaluation of the variances and covariances of order statistics in samples from the extreme value distribution. Annals of Mathematical Statistics 24, 282-287.

Lloyd, E. (1952). Least-squares estimation of location and scale parameters using order statistics. Biometrika 39(1/2), 88-95.

Mandal, S. and M. Samanta (2006). A large sample approximation of the o-blue of a location parameter. Journal of Statistical Theory and Applications 5(4), 343-350.

Mandal, S. and M. Samanta (2007). A unified approach to efficient estimation in simple linear regression. Sankhya 69(4), 635-647.

Miller, R. G. (1981). Survival Analysis. John Wiley \& Sons, New York.

Mukhopadhyay, N. (1950). Probability and statistical inference. Marcel Dekker.

Prescott, P. and A. T. Walden (1980). Maximum-likelihood estimation of the parameters of the generalized extreme-value distribution. Biometrika $67(3)$, $723-724$.

Rao, C. (1973). Linear Statistical Inference and Its Applications. Second Edition, John Wiley \& Sons, New York.

Raynal, J. and J. Salas (1986). Estimation procedure for the type-i extreme value distribution. Journal of Hydrology 87(3-4), 315-336.

Saha, S., M. Samanta, and S. Mandal (2014). Efficient estimation of parameters of the extreme value distribution. Sankhya B. Accepted, in press, doi:10.1007/s13571013-0074-3. 
Samanta, M. (1985). Efficient estimation of a location parameter by linear combinations of order statistics. Sankhya 47(1), 84-89.

Tawn, J. (1988). Bivariate extreme value theory: Models and estimation. Biometrika $75(3), 397-415$.

Wu, J. and P. Li (2003). Optimal parameter estimation of the extreme value distribution based on a type-ii censored sample. Communications in StatisticsTheory and Methods 3(32), 533-554. 